\title{
Intramolecular Silicon-Assisted Cross-Coupling: Total Synthesis of (+)-Brasilenyne
}

\author{
Scott E. Denmark* and Shyh-Ming Yang \\ Roger Adams Laboratory, Department of Chemistry, University of Illinois, \\ Urbana, Illinois 61801

\section{SUPPORTING INFORMATION}

\section{General Experimental}

All reactions were performed in oven-dried $\left(140{ }^{\circ} \mathrm{C}\right)$ or flame-dried glassware under an inert atmosphere of dry $\mathrm{Ar}$ or $\mathrm{N}_{2}$. The following reaction solvents were distilled from the indicated drying agents: diethyl ether ( $\mathrm{Na}$, benzophenone), THF ( $\mathrm{Na}$, benzophenone), $\mathrm{CH}_{2} \mathrm{Cl}_{2}$ $\left(\mathrm{P}_{2} \mathrm{O}_{5}\right)$, benzene $(\mathrm{Na})$, toluene $(\mathrm{Na})$, methanol $\left(\mathrm{Mg}(\mathrm{OMe})_{2}\right)$, triethylamine $\left(\mathrm{CaH}_{2}\right)$. $n$-Butyllithium solutions were titrated following the method of Gilman ${ }^{1}$. Brine refers to a saturated aqueous solution of $\mathrm{NaCl}$. Grignard solutions were titrated using 2,2'-phenanthroline as an indicator. ${ }^{2}$ Kugelrohr distillations were performed on a Büchi GKR-50 Kugelrohr; boiling points (bp) corresponding to uncorrected air-bath temperatures (ABT). All reaction temperatures correspond to internal temperatures measured by Teflon-coated thermocouples unless otherwise noted.

${ }^{1} \mathrm{H}$ NMR spectra and ${ }^{13} \mathrm{C}$ NMR spectra were recorded on a Varian Unity $400(400 \mathrm{MHz}$, $\left.{ }^{1} \mathrm{H} ; 100 \mathrm{MHz},{ }^{13} \mathrm{C}\right)$, Unity $500\left(500 \mathrm{MHz},{ }^{1} \mathrm{H} ; 126 \mathrm{MHz},{ }^{13} \mathrm{C}\right)$. Spectra are referenced to residual chloroform $\left(\delta 7.26 \mathrm{ppm},{ }^{1} \mathrm{H} ; \delta 77.0 \mathrm{ppm},{ }^{13} \mathrm{C}\right)$ and residual acetone $\left(\delta 2.04 \mathrm{ppm},{ }^{1} \mathrm{H} ; \delta\right.$ $\left.29.8 \mathrm{ppm},{ }^{13} \mathrm{C}\right)$. Chemical shifts are reported in ppm $(\delta)$; multiplicities are indicated by $\mathrm{s}$ (singlet), d (doublet), t (triplet), q (quartet), m (multiplet) and br (broad). Coupling constants, $J$, are reported in Hertz. Mass spectroscopy was performed by the University of Illinois Mass Spectrometer Center. Electron impact (EI) spectra were performed on a Finnigan-MAT CH-5 spectrometer. Data are reported in the form of $\mathrm{m} / \mathrm{z}$ (intensity relative to base peak $=100$ ). Infrared spectra (IR) were recorded on a Mattson Galaxy 5020 spectrophotometer. Peaks are reported in $\mathrm{cm}^{-1}$ with indicated relative intensities: $\mathrm{s}$ (strong, 67-100\%); m (medium, 34-66\%); w (weak, 0-33\%). Optical rotations were measured on Jasco DIP-360 polarimeter and were reported as follows: concentration $(\mathrm{c}=\mathrm{g} / 100 \mathrm{~mL})$, solvent. Elemental analyses were performed by the University of Illinois Microanalytical Service Laboratory.

Analytical thin-layer chromatography was performed on Merck silica or aluminum oxide, basic gel plates with QF-254 indicator. Visualization was accomplished with UV light and/or 
Iodide. Diethyl ether was of reagent grade and used as received; other solvents for chromatography and filtration were technical grade and distilled from the indicated drying agents: hexane and pentane $\left(\mathrm{CaCl}_{2}\right) ; \mathrm{CH}_{2} \mathrm{Cl}_{2}\left(\mathrm{CaCl}_{2}\right)$; ethyl acetate $\left(\mathrm{K}_{2} \mathrm{CO}_{3}\right)$. Column chromatography was performed using EM Science 230-400-mesh silica gel or Aldrich 150-mesh aluminum oxide, activated, basic, Brockmann I.

Analytical capillary gas chromatography (GC) was performed using the following gas chromatography fitted with a flame ionization detector $\left(\mathrm{H}_{2}\right.$ carrier gas, $\left.1 \mathrm{~mL} / \mathrm{min}\right)$ : Hewlett Packard 5890 Series II. The following column was used: HP-5 50-m cross-linked 5\%-Phenyl methyl silicone gum phase or Ultra-2 50-m cross-linked 5\%-Phenyl methyl silicone gum phase. The detector temperature was $300{ }^{\circ} \mathrm{C}$. Retention times $\left(t_{R}\right)$ and integrated ratios were obtained from Hewlett Packard 3393A integrators.

All commercial reagents were purified by distillation or recrystallization prior to use. A 1.0 M solution of tetrabutylammonium fluoride in THF was prepared from solid tetrabutylammonium fluoride trihydrate $\left(\mathrm{TBAF} \cdot 3 \mathrm{H}_{2} \mathrm{O}\right.$, Fluka) and distilled THF in a volumetric flask and was stored in a Schlenk bottle. $\pi$-Allylpalladium chloride dimer $[\text { allylPdCl }]_{2}$ (APC) was purchased from ACROS and was recrystallized from benzene prior to use. 
Scheme 1. Total Synthesis of (+)-Brasilenyne<smiles>C#[Y5]C#C[C@@H](CC)O[C@H]1CCOC1=O</smiles>

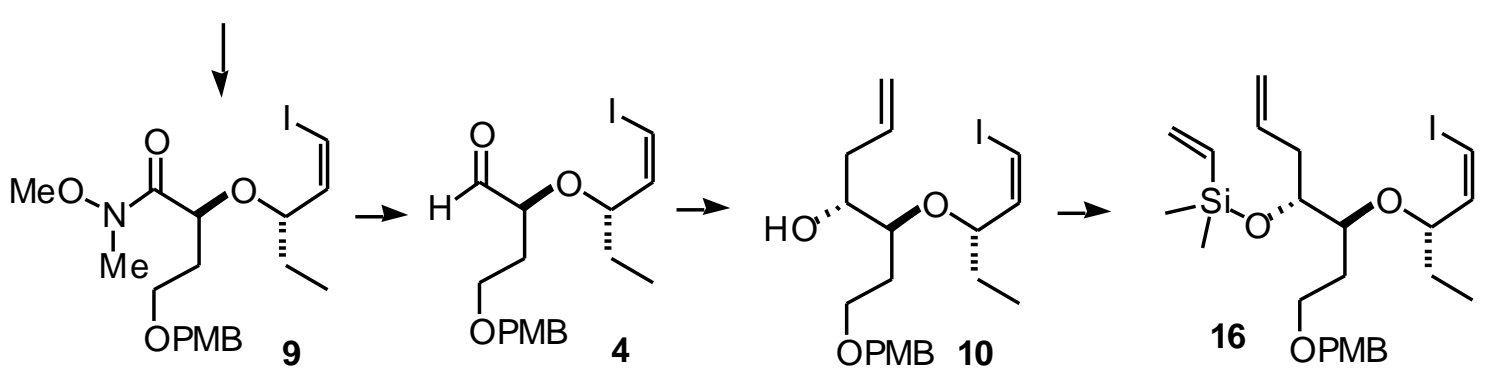

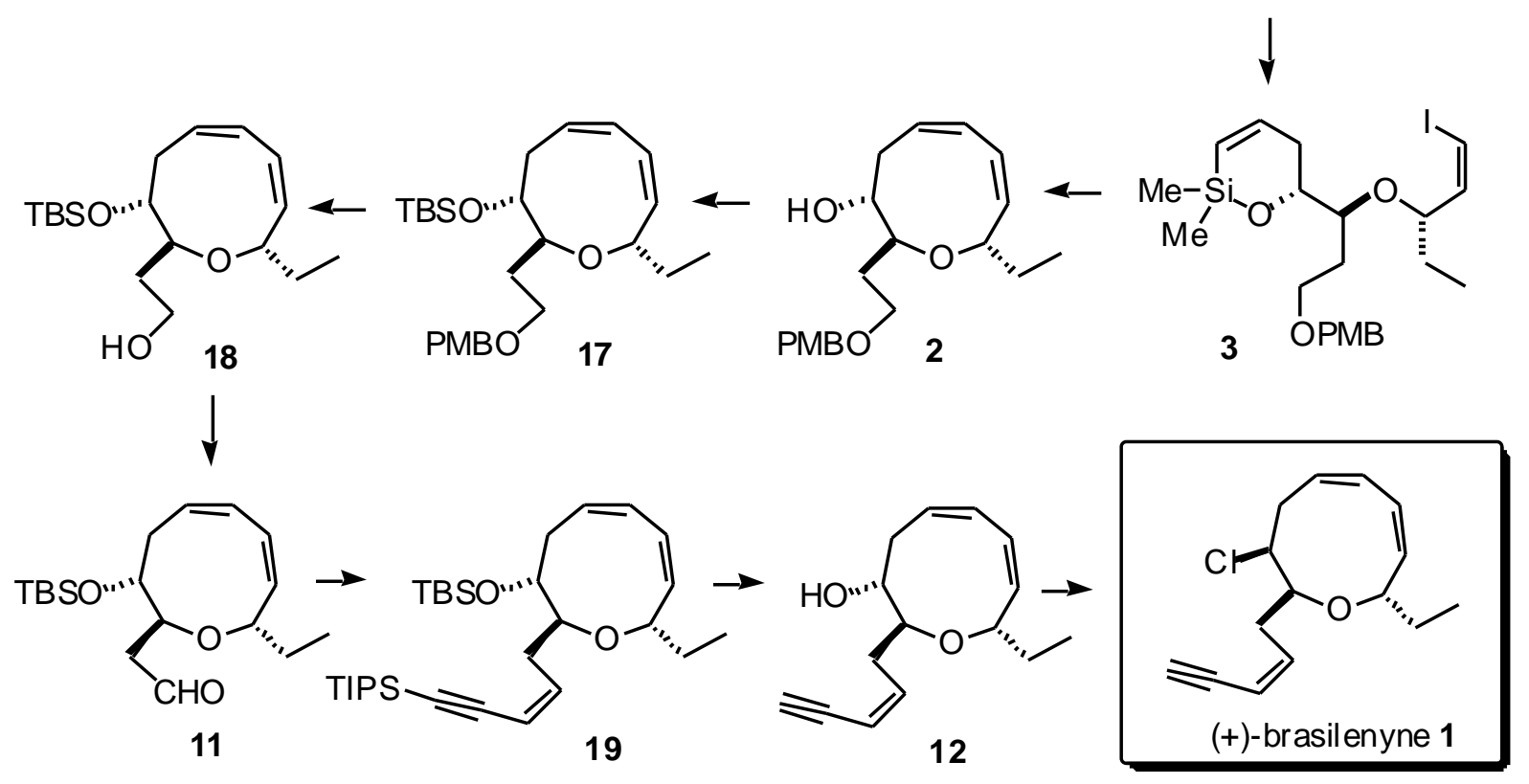




\section{Experimental Procedures}

\section{Preparation of 2-[(4S)-2-Ethyl-5-oxo-1,3-dioxolan-4-yl]acetic acid (13)}<smiles>O=C(O)C[C@H](O)C(=O)O</smiles>

L-malic acid

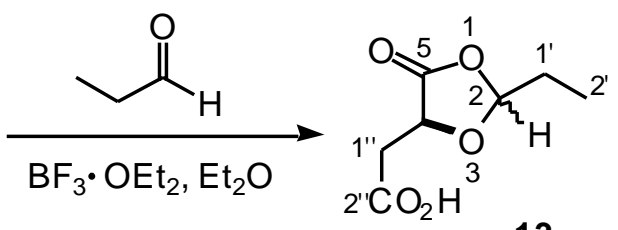

13

In a three-neck, $250 \mathrm{~mL}$ flask was placed L- $(S)$-malic acid $(6.7 \mathrm{~g}, 50 \mathrm{mmol}$, purchased from Aldrich, 99\% ee/CSP-GLC) and propanal (3.97 mL, $55 \mathrm{mmol}, 1.1$ equiv) in $\mathrm{Et}_{2} \mathrm{O}(100 \mathrm{~mL})$ under $\mathrm{N}_{2}$ at $-30{ }^{\circ} \mathrm{C} . \mathrm{BF}_{3} \bullet \mathrm{Et}_{2} \mathrm{O}(19.0 \mathrm{~mL}, 150 \mathrm{mmol}, 3.0$ equiv) was then added dropwise over 30 min. After complete addition of $\mathrm{BF}_{3} \bullet \mathrm{Et}_{2} \mathrm{O}$, the mixture was allowed to warm to $0{ }^{\circ} \mathrm{C}$ and was stirred for $1.5 \mathrm{~h}$. The mixture was then poured into $\mathrm{H}_{2} \mathrm{O}(100 \mathrm{~mL})$ and was separated. The aqueous layer was extracted with $\mathrm{Et}_{2} \mathrm{O}(2 \times 100 \mathrm{~mL})$. The combined organic layers were washed with brine $(2 \times 100 \mathrm{~mL})$, then was dried $\left(\mathrm{Na}_{2} \mathrm{SO}_{4}\right)$, and filtered. The ethereal solvent was removed by rotary evaporation and was then dried in vacuo to afford $7.4 \mathrm{~g}$ (85\%) of crude 13 (cis:trans $=81: 19$ ), which was directly subjected to next reaction without further purification. (This reaction can be run on a $200 \mathrm{mmol}$ scale in $84 \%$ yield.)

Analytical Data for 13 (cis:trans = 81:19):

${ }^{1} \underline{\mathrm{H} \mathrm{NMR}}: \quad\left(500 \mathrm{MHz}, \mathrm{CDCl}_{3}\right)$

(cis-isomer) 11.0 (br s, $1 \mathrm{H}, \mathrm{HO}), 5.52$ (td, $J=4.5,1.0,1 \mathrm{H}, \mathrm{HC}(2)), 4.62$ (ddd, $J$ $=7.0,4.0,1.0,1 \mathrm{H}, \mathrm{HC}(4)), 3.02\left(\mathrm{dd}, J=17.5,4.0,1 \mathrm{H}, \mathrm{HC}\left(1^{\prime}\right.\right.$ ' $\left.)\right), 2.84(\mathrm{dd}, J=$ 17.5, 7.0, $\left.1 \mathrm{H}, \mathrm{HC}\left(1^{\prime} ’\right)\right), 1.88-1.78\left(\mathrm{~m}, 2 \mathrm{H}, \mathrm{H}_{2} \mathrm{C}\left(1^{\prime}\right)\right), 0.99$ (t, $J=7.5,3 \mathrm{H}$, $\mathrm{H}_{3} \mathrm{C}\left(2^{\prime}\right)$

(trans-isomer) 11.0 (br s, $1 \mathrm{H}, \mathrm{HO}), 5.68(\mathrm{td}, J=5.0,1.5,1 \mathrm{H}, \mathrm{HC}(2)), 4.65$ (ddd, $J=5.5,4.0,1.5,1 \mathrm{H}, \mathrm{HC}(4)), 2.96\left(\mathrm{dd}, J=17.5,4.0,1 \mathrm{H}, \mathrm{HC}\left(1^{\prime}\right.\right.$ ') $), 2.91$ (dd, $J=$ 17.5, 6.0, $\left.1 \mathrm{H}, \mathrm{HC}\left(1^{\prime} ’\right)\right), 1.88-1.78\left(\mathrm{~m}, 2 \mathrm{H}, \mathrm{H}_{2} \mathrm{C}\left(1^{\prime}\right)\right), 0.98(\mathrm{t}, J=7.5,3 \mathrm{H}$, $\mathrm{H}_{3} \mathrm{C}\left(2^{\prime}\right)$

${ }^{13} \mathrm{C} \mathrm{NMR}: \quad\left(126 \mathrm{MHz}, \mathrm{CDCl}_{3}\right)$

(cis-isomer) 175.2 (C(2’’)), 172.2 (C(5)), $105.6(\mathrm{C}(2)), 71.1(\mathrm{C}(4)), 35.3\left(\mathrm{C}\left(1^{\prime \prime}\right)\right)$, $26.8\left(\mathrm{C}\left(1^{\prime}\right)\right), 6.7\left(\mathrm{C}\left(2^{\prime}\right)\right)$

(trans-isomer) 175.5 (C(2'’)), 172.1 (C(5)), 106.8 (C(2)), $70.1(\mathrm{C}(4)), 35.5$ $\left(\mathrm{C}\left(1^{\prime \prime}\right)\right), 26.9\left(\mathrm{C}\left(1^{\prime}\right)\right), 6.7\left(\mathrm{C}\left(2^{\prime}\right)\right)$ 


\section{Preparation of 2-[(4S)-2-Ethyl-5-oxo-1,3-dioxolan-4-yl]ethanol (14)}
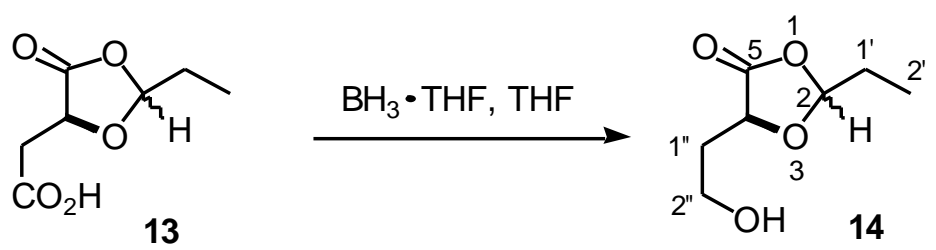

In a three-neck, $250 \mathrm{~mL}$ flask was placed $13(7.4 \mathrm{~g}, 42.5 \mathrm{mmol})$ in THF (40 mL) under $\mathrm{N}_{2}$ at $0{ }^{\circ} \mathrm{C} . \mathrm{BH}_{3} \bullet \mathrm{THF}$ (0.55 in THF, $85 \mathrm{~mL}, 46.75 \mathrm{mmol}, 1.1$ equiv) was then added dropwise with gentle bubbling was observed. After complete addition of $\mathrm{BH}_{3} \bullet \mathrm{THF}$, the mixture was stirred at $0{ }^{\circ} \mathrm{C}$ for $3 \mathrm{~h}$. $\mathrm{MeOH}(40 \mathrm{~mL})$ was then added dropwise at $0{ }^{\circ} \mathrm{C}$. After complete addition of $\mathrm{MeOH}$, the mixture was directly concentrated. The residue was dissolved in $\mathrm{CH}_{2} \mathrm{Cl}_{2}$ $(50 \mathrm{~mL}) / \mathrm{H} 2 \mathrm{O}(50 \mathrm{~mL})$ and was separated. The aqueous layer was extracted with $\mathrm{CH}_{2} \mathrm{Cl}_{2}(3 \times 50$ $\mathrm{mL})$. The combined organic layers were dried $\left(\mathrm{Na}_{2} \mathrm{SO}_{4}\right)$, and filtered. The solvent was removed by rotary evaporation and was then dried in vacuo to afford $5.6 \mathrm{~g}(82 \%)$ of crude 14 (cis:trans= $83: 17$ ), which was directly subjected to next reaction without further purification. (This reaction can be run on a 168 mmol scale in $82 \%$ yield.)

Analytical Data for 14 (cis:trans = 83:17):

${ }^{1} \underline{\mathrm{H} N M R}: \quad\left(500 \mathrm{MHz}, \mathrm{CDCl}_{3}\right)$

(cis-isomer) 5.51 (td, $J=4.5,1.0,1 \mathrm{H}, \mathrm{HC}(2)), 4.45$ (ddd, $J=7.0,4.5,1.0,1 \mathrm{H}$, $\mathrm{HC}(4))$, 3.89-3.80 (m, $2 \mathrm{H}, \mathrm{H}_{2} \mathrm{C}\left(2^{\prime} ’\right)$ ), 2.19 (dtd, $J=14.5,7.5,5.0,1 \mathrm{H}, \mathrm{HC}\left(1^{\prime \prime}\right)$ ), 2.04(ddt, $J=14.5,7.0,5.0,1 \mathrm{H}, \mathrm{HC}\left(1^{\prime} ’\right)$ ), 1.87 (qd, $J=7.5,4.5,2 \mathrm{H}, \mathrm{H}_{2} \mathrm{C}\left(1^{\prime}\right)$ ), 1.85 (br s, $1 \mathrm{H}, \mathrm{HO}$ ), 1.02 (t, $J=7.5,3 \mathrm{H}, \mathrm{H}_{3} \mathrm{C}\left(2^{\prime}\right.$ )

(trans-isomer) $5.67(\mathrm{td}, J=5.0,1.0,1 \mathrm{H}, \mathrm{HC}(2)), 4.55(\mathrm{ddd}, J=7.0,5.0,1.0,1 \mathrm{H}$, $\mathrm{HC}(4))$, 3.89-3.80 (m, $\left.2 \mathrm{H}, \mathrm{H}_{2} \mathrm{C}(1)\right)$, 2.05-2.00 (m, $2 \mathrm{H}, \mathrm{H}_{2} \mathrm{C}\left(1^{\prime}\right.$ ')), 1.83 (qd, $J=$ 7.5, 4.5, $2 \mathrm{H}, \mathrm{H}_{2} \mathrm{C}\left(1^{\prime}\right.$ )), 1.85 (br s, $\left.1 \mathrm{H}, \mathrm{HO}\right), 1.00$ (t, $J=7.5,3 \mathrm{H}, \mathrm{H}_{3} \mathrm{C}\left(2^{\prime}\right)$

${ }^{13} \mathrm{C} \mathrm{NMR}: \quad\left(126 \mathrm{MHz}, \mathrm{CDCl}_{3}\right)$

(cis-isomer) $173.8(\mathrm{C}(5)), 105.3(\mathrm{C}(2)), 79.2$ (C(4)), 58.6 (C(2’’)), $33.3\left(\mathrm{C}\left(1^{\prime \prime}\right)\right)$, $26.9\left(\mathrm{C}\left(1^{\prime}\right)\right), 6.7\left(\mathrm{C}\left(2^{\prime}\right)\right)$

(trans-isomer) 174.0 (C(5)), 106.1(C(2)), 71.7(C(4)), $58.4\left(\mathrm{C}\left(2^{\prime}{ }^{\prime \prime}\right)\right), 33.0\left(\mathrm{C}\left(1^{\prime \prime}\right)\right)$, $27.8\left(\mathrm{C}\left(1^{\prime}\right)\right), 6.8\left(\mathrm{C}\left(2^{\prime}\right)\right)$ 
Preparation of

dimethylsilane (6)

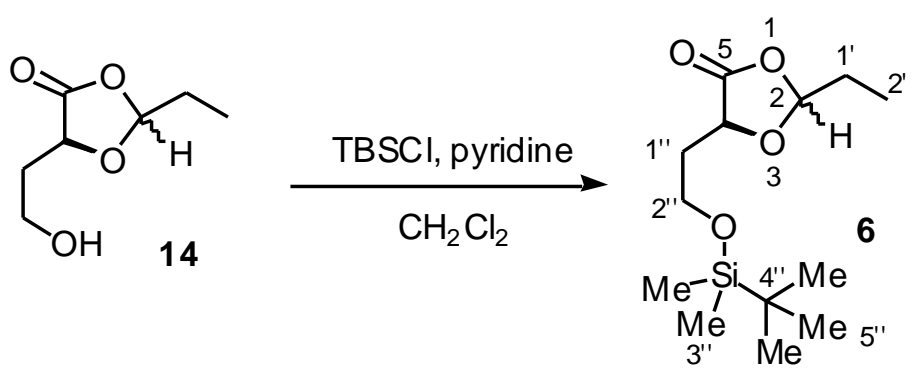

In a three-neck, $100 \mathrm{~mL}$ flask was placed $t$-butylchlorodimethylsilane $(7.91 \mathrm{~g}, 52.5$ mmol, 1.5 equiv) in $\mathrm{CH}_{2} \mathrm{Cl}_{2}(20 \mathrm{~mL})$ under $\mathrm{N}_{2}$ at room temperature. Pyridine $(8.48 \mathrm{~mL}, 105$ mmol, 3.0 equiv) was then added dropwise. The mixture was stirred for $10 \mathrm{~min}$ and a solution of 14 (5.6 g, $35.0 \mathrm{mmol})$ in $\mathrm{CH}_{2} \mathrm{Cl}_{2}(15 \mathrm{~mL})$ was added dropwise. The mixture was stirred for $4 \mathrm{~h}$ whereupon a white suspension was obtained. The mixture was poured into $\mathrm{H}_{2} \mathrm{O}(35 \mathrm{~mL})$ and the aqueous layer was extracted with $\mathrm{CH}_{2} \mathrm{Cl}_{2}(3 \times 35 \mathrm{~mL})$. The combined organic layers were dried $\left(\mathrm{Na}_{2} \mathrm{SO}_{4}\right)$, and filtered. The solvent was removed by rotary evaporation to give crude product, which was purified by chromatography (silica gel, hexane/EtOAc, $1 / 0$ to 19/1) followed by Kugelrohr distillation to afford $8.15 \mathrm{~g}(85 \%)$ of $\mathbf{6}$ (cis:trans $=83: 17)$ as a colorless liquid. (This reaction can be run on a $138 \mathrm{mmol}$ scale in $83 \%$ yield.)

Analytical Data for 6 (cis:trans $=84: 16$ ):

bp: $\quad 110-115^{\circ} \mathrm{C}(0.1 \mathrm{mmHg}, \mathrm{ABT})$

${ }^{1}$ H NMR: $\quad\left(500 \mathrm{MHz}, \mathrm{CDCl}_{3}\right)$

(cis-isomer) $5.48(\mathrm{td}, J=4.5,1.0,1 \mathrm{H}, \mathrm{HC}(2)), 4.42(\mathrm{ddd}, J=8.5,4.5,1.0,1 \mathrm{H}$, $\mathrm{HC}(4)), 3.83$ (dt, $J=10.5,5.0,1 \mathrm{H}, \mathrm{HC}\left(2^{\prime}{ }^{\prime}\right)$ ), 3.74 (ddd, $J=10.5,8.0,5.0,1 \mathrm{H}$, HC(2'”)), 2.11 (dddd, $J=14.0,8.0,5.5,4.0,1 \mathrm{H}, \mathrm{HC}\left(1^{\prime}\right.$ ')), 1.92-1.78 (m, $3 \mathrm{H}$, $\mathrm{HC}\left(1^{\prime}\right.$ '), $\left.\mathrm{H}_{2} \mathrm{C}\left(1^{\prime}\right)\right), 1.00$ (t, $J=7.5,3 \mathrm{H}, \mathrm{H}_{3} \mathrm{C}\left(2^{\prime}\right), 0.88$ (s, $9 \mathrm{H}, 3$ x $\mathrm{H}_{3} \mathrm{C}\left(5^{\prime}\right.$ ') ), 0.05 (s, $6 \mathrm{H}, 2 \times \mathrm{H}_{3} \mathrm{C}\left(3^{\prime \prime}\right)$ )

(trans-isomer) $5.62(\mathrm{td}, J=4.5,1.0,1 \mathrm{H}, \mathrm{HC}(2)), 4.51(\mathrm{ddd}, J=6.0,5.0,1.0,1 \mathrm{H}$, $\mathrm{HC}(4))$, 3.84-3.80 (m, $1 \mathrm{H}, \mathrm{HC}\left(2^{\prime}\right.$ ') ), 3.75-3.71 (m, $1 \mathrm{H}, \mathrm{HC}\left(2^{\prime}\right.$ ')), 2.00-1.95 (m, $\left.2 \mathrm{H}, \mathrm{H}_{2} \mathrm{C}\left(1^{\prime \prime}\right)\right)$ ), 1.90-1.78 (m, $\left.2 \mathrm{H}, \mathrm{H}_{2} \mathrm{C}\left(1^{\prime}\right)\right), 0.99$ (t, $J=7.5,3 \mathrm{H}, \mathrm{H}_{3} \mathrm{C}\left(2^{\prime}\right), 0.87$ (s, $\left.9 \mathrm{H}, 3 \times \mathrm{H}_{3} \mathrm{C}\left(5^{\prime},\right)\right), 0.05$ (s, $6 \mathrm{H}, 2 \times \mathrm{H}_{3} \mathrm{C}\left(3^{\prime}\right.$ ') )

${ }^{13} \mathrm{C} \mathrm{NMR}: \quad\left(126 \mathrm{MHz}, \mathrm{CDCl}_{3}\right)$

(cis-isomer) $173.8(\mathrm{C}(5)), 105.0(\mathrm{C}(2)), 71.6(\mathrm{C}(4)), 58.2$ (C(2’’)), 33.9 (C(1'))), $27.0\left(\mathrm{C}\left(1^{\prime}\right)\right), 25.8$ (3 x C $\left.\left(5^{\prime \prime}\right)\right), 18.2\left(\mathrm{C}\left(4^{\prime \prime}\right)\right), 6.7\left(\mathrm{C}\left(2^{\prime}\right)\right),-5.5\left(\mathrm{C}\left(3^{\prime \prime}\right)\right),-5.6$ $\left(\mathrm{C}\left(3^{\prime \prime}\right)\right)$ 
(trans-isomer) $173.9(\mathrm{C}(5)), 105.9(\mathrm{C}(2)), 70.8 \quad(\mathrm{C}(4)), 58.1 \quad\left(\mathrm{C}\left(2^{\prime \prime}\right)\right), 33.4$ $\left(\mathrm{C}\left(1^{\prime \prime}\right)\right), 27.8\left(\mathrm{C}\left(1^{\prime}\right)\right), 25.8$ (3 x C(5'’)), 18.2 (C(4'’)), $6.8\left(\mathrm{C}\left(2^{\prime}\right)\right),-5.56\left(\mathrm{C}\left(3^{\prime \prime}\right)\right)$, $5.63\left(\mathrm{C}\left(3^{\prime},\right)\right)$

IR: $\quad(\mathrm{NaCl})$

2954 (s), 2931 (s), 2885 (s), 1803 (s), 1739 (m), 1467 (m), 1409 (m), 1255 (s), 1199 (s), 1106 (s), 1008 (“m), 954 (s), 838 (s), 777 (s)

MS: $\quad(\mathrm{EI}, 70 \mathrm{eV})$

$275\left(59, \mathrm{M}^{+}+1\right), 274\left(\mathrm{M}^{+}, 1\right), 259$ (18), 245 (10), 217 (100), 173 (8), 131 (72), 115 (19), 101 (10)

TLC: $\quad R_{f} 0.30$ (silica gel, hexane/EtOAc, 19/1, PMA)

Analysis: $\quad \mathrm{C}_{13} \mathrm{H}_{26} \mathrm{O}_{4} \mathrm{Si} \quad$ (274.44)

Calculated: $\quad$ C, $56.90 ; \quad \mathrm{H}, 9.55 \%$

Found: $\quad$ C, $56.84 ; \quad H, 9.71 \%$

\section{Preparation of (3S)-\{[(1S)-1-Ethyl-3-trimethylsilylpropynyl]oxy $\}$ tetrahydrofuran-2-one (5)}

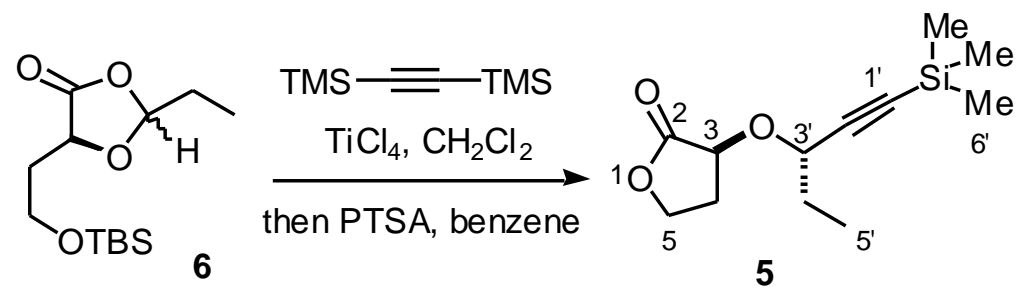

In a three-neck, $250 \mathrm{~mL}$ flask was placed $6(5.48 \mathrm{~g}, 20.0 \mathrm{mmol}, 1.5$ equiv) and bis(trimethylsilyl)acetylene (6.8 g, $40.0 \mathrm{mmol}, 2.0$ equiv) in $\mathrm{CH}_{2} \mathrm{Cl}_{2}(100 \mathrm{~mL})$ under $\mathrm{N}_{2}$ at -72 ${ }^{\circ} \mathrm{C}$. A freshly distilled $\mathrm{TiCl}_{4}(3.07 \mathrm{~mL}, 28.0 \mathrm{mmol}, 1.4$ equiv) was then added in one portion. The resulting yellow solution was stirred at $-72{ }^{\circ} \mathrm{C}$ for $3 \mathrm{~h}$ and $\mathrm{MeOH}(40 \mathrm{~mL})$ was added. The mixture was allowed to warm to room temperature and was stirred for $30 \mathrm{~min}$. The mixture was then directly concentrated and the residue was dissolved in $\mathrm{CH}_{2} \mathrm{Cl}_{2}(50 \mathrm{~mL}) / \mathrm{H}_{2} \mathrm{O}(50 \mathrm{~mL})$. The aqueous layer was extracted with $\mathrm{CH}_{2} \mathrm{Cl}_{2}(3 \times 50 \mathrm{~mL})$. The combined organic layers were dried $\left(\mathrm{Na}_{2} \mathrm{SO}_{4}\right)$, and filtered. After removal of solvent, the residue was dissolved in benzene $(50 \mathrm{~mL})$. p-Toulenesulfonic acid monohydrate ( $38 \mathrm{mg}, 0.02 \mathrm{mmol}, 0.01$ equiv) was then added and the mixture was heated to reflux for $1 \mathrm{~h}$ in a Dean-Stark apparatus. After cooling, the solvent was removed by rotary evaporation to give a crude product, which was purified by chromatography ( silica gel, hexane/EtOAc, 19/1 to 9/1) followed by Kugelrohr distillation to afford $4.13 \mathrm{~g}$ (86\%) of 5 as a white solid. (This reaction can be run on a $40.0 \mathrm{mmol}$ scale in $83 \%$ yield.) 
Analytical Data for 5:

bp: $\quad 105-110^{\circ} \mathrm{C}(0.3 \mathrm{mmHg}, \mathrm{ABT})$

1․

$4.62\left(\mathrm{t}, J=7.0,1 \mathrm{H}, \mathrm{HC}\left(3^{\prime}\right)\right), 4.48(\mathrm{ddd}, J=8.0,1 \mathrm{H}, \mathrm{HC}(3)), 4.39(\mathrm{td}, J=9.0$, 4.0, $1 \mathrm{H}, \mathrm{HC}(5)$ ), 4.24 (ddd, $J=9.0,8.5,6.5,1 \mathrm{H}, \mathrm{HC}(5)$ ), 2.55 (dddd, $J=13.0$, 8.0, 7.0, 4.0, $1 \mathrm{H}, \mathrm{HC}(4)), 2.29$ (dq, $J=13.0,8.5,1 \mathrm{H}, \mathrm{HC}(4)), 1.80-1.66$ (m, $2 \mathrm{H}$, $\left.\mathrm{H}_{2} \mathrm{C}\left(4^{\prime}\right)\right), 0.99$ (t, $J=7.5,3 \mathrm{H}, \mathrm{H}_{3} \mathrm{C}\left(5^{\prime}\right), 0.17$ (s, 9 H, 3 x $\mathrm{H}_{3} \mathrm{C}\left(6^{\prime}\right)$ )

${ }^{13} \mathrm{C} \mathrm{NMR}: \quad\left(126 \mathrm{MHz}, \mathrm{CDCl}_{3}\right)$

$175.4(\mathrm{C}(2)), 103.7\left(\mathrm{C}\left(1^{\prime}\right)\right), 91.4\left(\mathrm{C}\left(2^{\prime}\right)\right), 70.98,70.95,65.4(\mathrm{C}(5)), 30.1(\mathrm{C}(4))$, $28.7\left(\mathrm{C}\left(4^{\prime}\right)\right), 9.4\left(\mathrm{C}\left(5^{\prime}\right)\right),-0.2\left(3 \times \mathrm{C}\left(6^{\prime}\right)\right)$

IR: $\quad(\mathrm{NaCl})$

2966 (s), 2894 (m), 2173 (m), 1764 (s), 1450 (m), 1384 (m), 1294 (m), 1249 (s), 1216 (s), 1180 (s), 1128 (s), 1105 (s), 1022 (s), 968 (s), 881 (m), 842 (s), 761 (s)

MS: $\quad(\mathrm{EI}, 70 \mathrm{eV})$

$240\left(1, \mathrm{M}^{+}\right), 225$ (8), 211 (18), 183 (6), 158 (30), 155 (58), 139 (16), 131 (25), 123 (26), 109 (35), 97 (100), 86 (66), 73 (24)

Opt. Rot.: $\quad[\alpha]_{\mathrm{D}}{ }^{24}-138.6\left(c=1.08, \mathrm{CHCl}_{3}\right)$

TLC: $\quad R_{f} 0.15$ (silica gel, hexane/EtOAc, 19/1, PMA)

Analysis: $\quad \mathrm{C}_{12} \mathrm{H}_{20} \mathrm{O}_{3} \mathrm{Si} \quad(240.38)$

$\begin{array}{lll}\text { Calculated: } & \text { C, 59.96; } & \text { H, 8.39\% } \\ \text { Found: } & \text { C, 59.72; } & \text { H, 8.39\% }\end{array}$

\section{Preparation of (3S)-\{[(1S)-1-Ethyl-3-iodopropynyl]oxy\}tetrahydrofuran-2-one (7)}

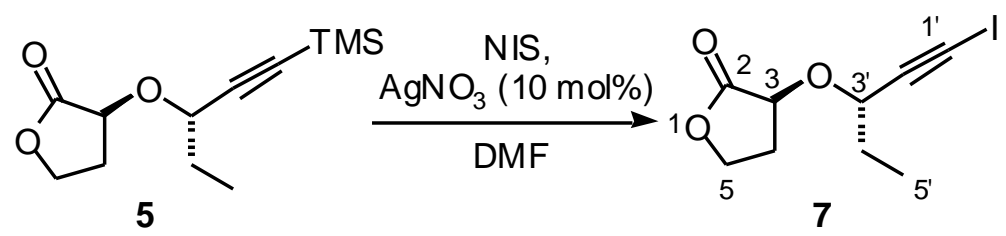

In a three-neck, $100 \mathrm{~mL}$ flask was placed 5 (2.4 g, $10.0 \mathrm{mmol}, 1.5$ equiv) in DMF (20 $\mathrm{mL}$ ) under $\mathrm{N}_{2}$ at room temperature. $N$-Iodosuccinimide $(2.7 \mathrm{~g}, 12.0 \mathrm{mmol}, 1.2$ equiv) and $\mathrm{AgNO}_{3}(170 \mathrm{mg}, 1.0 \mathrm{mmol}, 0.1$ equiv) was then added sequentially. The mixture was stirred at room temperature for $10 \mathrm{~min}$ and $\mathrm{Et}_{2} \mathrm{O}(50 \mathrm{~mL})$ was added. The mixture was washed with saturated aqueous $\mathrm{NH}_{4} \mathrm{Cl}$ solution $(2 \times 25 \mathrm{~mL})$. The aqueous layer was extracted with $\mathrm{Et}_{2} \mathrm{O}(25$ $\mathrm{mL})$. The combined organic layers were washed with brine $(30 \mathrm{~mL})$, then were dried $\left(\mathrm{Na}_{2} \mathrm{SO}_{4}\right)$, 
and filtered. The solvent was removed by rotary evaporation to give crude product, which was purified by chromatography (silica gel, hexane/EtOAc, 19/1 to 4/1) to afford $2.8 \mathrm{~g}(95 \%)$ of 7 as a pale yellow oil. (This reaction can be run on a 40.0 mmol scale in $93 \%$ yield.) Analytical Data for 7:

${ }^{1} \underline{\mathrm{H} \mathrm{NMR}}: \quad\left(500 \mathrm{MHz}, \mathrm{CDCl}_{3}\right)$

$4.80\left(\mathrm{t}, J=7.5,1 \mathrm{H}, \mathrm{HC}\left(3^{\prime}\right)\right), 4.46(\mathrm{t}, J=8.0,1 \mathrm{H}, \mathrm{HC}(3)), 4.39(\mathrm{td}, J=8.5,4.0,1$ H, HC(5)), 4.24 (td, $J=8.5,7.0,1 \mathrm{H}, \mathrm{HC}(5)), 2.59-2.53$ (m, $1 \mathrm{H}, \mathrm{HC}(4)), 2.28$ (dq, $J=13.5,8.5,1 \mathrm{H}, \mathrm{HC}(4)), 1.79-1.69$ (m, $\left.2 \mathrm{H}, \mathrm{H}_{2} \mathrm{C}\left(4^{\prime}\right)\right), 0.99$ (t, $J=7.5,3 \mathrm{H}$, $\left.\mathrm{H}_{3} \mathrm{C}\left(5^{\prime}\right)\right)$

${ }^{13}$ C NMR: $\quad\left(100 \mathrm{MHz}, \mathrm{CDCl}_{3}\right)$

175.3 (C(2)), 92.9 (C(2’)), 71.7, 71.0, 65.4 (C(5)), 29.9 (C(4)), 28.9 (C(4')), 9.4 $\left(\mathrm{C}\left(5^{\prime}\right)\right), 9.4\left(\mathrm{C}\left(1^{\prime}\right)\right)$

IR: $\quad(\mathrm{NaCl})$

2971 (s), 2937 (s), 2877 (s), 2179 (m), 1776 (s), 1461 (s), 1382 (s), 1326 (s), 1220

(s), 1180 (s), 1130 (s), 1006 (s), 948 (s), 889 (m), 808 (m), 715 (m)

MS: $\quad(\mathrm{EI}, 70 \mathrm{eV})$

295 (21, M+ + 1), 265 (10), 237 (3), 209 (4), 193 (48), 167 (41), 143 (11), 103

(100), 85 (37), 83 (45), 66 (53)

Opt. Rot.: $\quad[\alpha]_{\mathrm{D}}{ }^{24}-122.7\left(c=1.17, \mathrm{CHCl}_{3}\right)$

TLC: $\quad R_{f} 0.21$ (silica gel, hexane/EtOAc, 4/1, PMA)

Analysis: $\quad \mathrm{C}_{9} \mathrm{H}_{11} \mathrm{O}_{3} \mathrm{I} \quad$ (294.09)

Calculated: $\quad$ C, 36.76; $\quad$ H, 3.77, I, 43.15\%

Found: $\quad$ C, $36.50 ; \quad H, 3.69, \quad$ I, $42.88 \%$

Preparation of (3S)-\{[(1S,2Z)-1-Ethyl-3-iodo-2-propenyl]oxy $\}$ tetrahydrofuran-2-one (8)
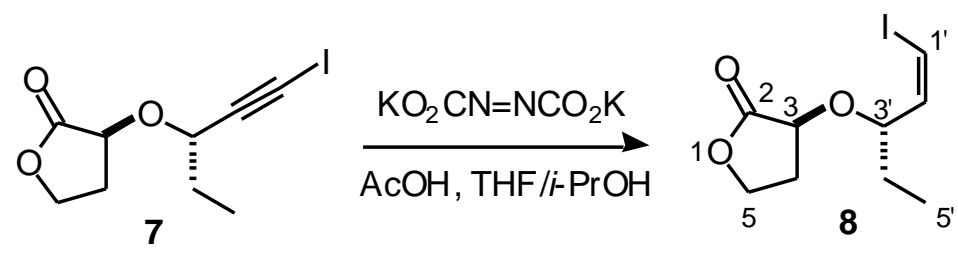

In a three-neck, $50 \mathrm{~mL}$ flask was placed $7(3.53 \mathrm{~g}, 12 \mathrm{mmol})$ and potassium azodicarboxylate $\left(\mathrm{KO}_{2} \mathrm{CN}=\mathrm{NCO}_{2} \mathrm{~K}\right)(3.49 \mathrm{~g}, 18 \mathrm{mmol}, 1.5$ equiv $)$ in $\mathrm{THF}(12 \mathrm{~mL}) / i-\mathrm{PrOH}(12$ $\mathrm{mL}$ ) under $\mathrm{N}_{2}$ at room temperature. Acetic acid $(1.97 \mathrm{~mL}, 36 \mathrm{mmol}, 3.0$ equiv) was then added slowly by syringe pump over $1 \mathrm{~h}$. After complete addition of acetic acid, the mixture was stirred 
for $1 \mathrm{~h}$ and additional potassium azodicarboxylate (3.49 g, $18 \mathrm{mmol}, 1.5$ equiv). Again, acetic acid (1.97 mL, $36 \mathrm{mmol}, 3.0$ equiv) was then added slowly by syringe pump over $1 \mathrm{~h}$. After complete addition of acetic acid, the mixture was stirred for $1 \mathrm{~h}$ and additional potassium azodicarboxylate (1.16 g, $6 \mathrm{mmol}, 0.5$ equiv) and acetic acid ( $0.68 \mathrm{~mL}, 12 \mathrm{mmol}, 1.0$ equiv) were added sequentially by a procedure described above. The mixture was then poured into a $250 \mathrm{~mL}$ beaker together with $\mathrm{Et}_{2} \mathrm{O}(20 \mathrm{~mL})$ and aqueous $\mathrm{HCl}$ solution $(3.0 \mathrm{M}, 20 \mathrm{~mL})$. The aqueous layer was extracted with $\mathrm{Et}_{2} \mathrm{O}(3 \times 20 \mathrm{~mL})$. The combined organic layers were washed with brine $(2 \times 20 \mathrm{~mL})$, then was dried $\left(\mathrm{Na}_{2} \mathrm{SO}_{4}\right)$, and filtered. After removal of solvent, the residue was treated with pyridine $(5 \mathrm{~mL})$ and the mixture was stirred at room temperature for 12 $\mathrm{h}$ to remove the small amount of over reduction product. The mixture was dissolved in $\mathrm{CH}_{2} \mathrm{Cl}_{2}$ $(20 \mathrm{~mL})$ which was washed with aqueous $\mathrm{HCl}$ solution $(3.0 \mathrm{M}, 2 \times 20 \mathrm{~mL})$. The combined aqueous layers were extracted with $\mathrm{CH}_{2} \mathrm{Cl}_{2}(2 \times 20 \mathrm{~mL})$. The combined organic layers were dried $\left(\mathrm{Na}_{2} \mathrm{SO}_{4}\right)$, and filtered. The solvent was removed by rotary evaporation to give a crude product, which was purified by chromatography (silica gel, hexane/EtOAc, 19/1 to 4/1) followed by Kugelrohr distillation to afford $2.84 \mathrm{~g}(80 \%)$ of $\mathbf{8}$ as a pale yellow oil. (This reaction can be run on a 40.0 mmol scale in $78 \%$ yield.)

Analytical Data for 8:

bp: $\quad 155-160{ }^{\circ} \mathrm{C}(0.3 \mathrm{mmHg}, \mathrm{ABT})$

${ }^{1} \underline{\mathrm{H} \mathrm{NMR}}: \quad\left(500 \mathrm{MHz}, \mathrm{CDCl}_{3}\right)$

$6.54\left(\mathrm{dd}, J=8.0,1.0,1 \mathrm{H}, \mathrm{HC}\left(1^{\prime}\right)\right), 6.13$ (t, $\left.J=8.0,1 \mathrm{H}, \mathrm{HC}\left(2^{\prime}\right)\right), 4.58$ (q, $J=7.0$, $\left.1 \mathrm{H}, \mathrm{HC}\left(3^{\prime}\right)\right), 4.37(\mathrm{td}, J=9.0,3.5,1 \mathrm{H}, \mathrm{HC}(5)), 4.19(\mathrm{td}, J=9.0,6.5,1 \mathrm{H}$, $\mathrm{HC}(5)), 4.15$ (t, $J=8.0,1 \mathrm{H}, \mathrm{HC}(3)), 2.45$ (dddd, $J=13.0,8.0,6.5,3.5,1 \mathrm{H}$, $\mathrm{HC}(4)$ ), 2.21 (dq, $J=13.0,9.0,1 \mathrm{H}, \mathrm{HC}(4)$ ), 1.72 (d quint, $J=14.5,7.5,1 \mathrm{H}$, $\mathrm{HC}\left(4^{\prime}\right)$ ), 1.57 (d quint, $J=14.5,7.5,1 \mathrm{H}, \mathrm{HC}\left(4^{\prime}\right)$ ), 0.97 (t, $J=7.5,3 \mathrm{H}, \mathrm{H}_{3} \mathrm{C}\left(5^{\prime}\right)$ )

${ }^{13}$ C NMR: $\quad\left(126 \mathrm{MHz}, \mathrm{CDCl}_{3}\right)$

$175.2(\mathrm{C}(2)), 141.0\left(\mathrm{C}\left(2^{\prime}\right)\right), 85.2\left(\mathrm{C}\left(1^{\prime}\right)\right), 82.8\left(\mathrm{C}\left(3^{\prime}\right)\right), 71.4(\mathrm{C}(3)), 65.0(\mathrm{C}(5))$, 30.3 (C(4)), 27.4 (C(4')), 9.3 (C(5'))

IR: $\quad(\mathrm{NaCl})$

3068 (m), 2967 (m), 2933 (m), 2879 (m), 1779 (s), 1608 (m), 1461 (m), 1382 (m), 1332 (m), 1274 (m), 1220 (m), 1176 (s), 1130 (s), 1024 (s), 948 (m), 709 (m)

MS: $\quad(\mathrm{EI}, 70 \mathrm{eV})$

$297\left(54, \mathrm{M}^{+}+1\right), 267$ (26), 239 (2), 195 (100), 169 (65), 153 (21), 103 (59), 86 (13), 68 (13)

Opt. Rot.: $\quad[\alpha]_{\mathrm{D}}{ }^{24}-16.3\left(c=1.06, \mathrm{CHCl}_{3}\right)$

TLC: $\quad R_{f} 0.17$ (silica gel, hexane/EtOAc, 4/1, PMA) 
Analysis: $\quad \mathrm{C}_{9} \mathrm{H}_{13} \mathrm{O}_{3} \mathrm{I} \quad(296.11)$

$\begin{array}{llll}\text { Calculated: } & \text { C, 36.51; } & \text { H, 4.43, } & \text { I, } 42.86 \% \\ \text { Found: } & \text { C, 36.34; } & \text { H, 4.17, } & \text { I, } 42.58 \%\end{array}$

\section{Preparation of (2 $S)$ - $N$-Methoxy- $N$-methyl-2- $\{[(1 S, 2 Z)-1-e t h y l-3-i o d o-2-p r o p e n y l] o x y\}-4-$ hydroxybutanamide (15)}

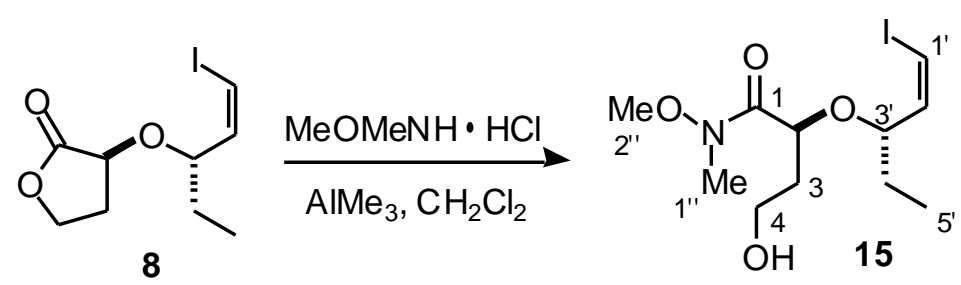

In a three-neck, $100 \mathrm{~mL}$ flask was placed $\mathrm{N}, \mathrm{O}$-dimethylhydroxylamine hydrochloride salt (1.76 g, $18.0 \mathrm{mmol}, 2.0$ equiv) in $\mathrm{CH}_{2} \mathrm{Cl}_{2}(10 \mathrm{~mL})$ under $\mathrm{N}_{2}$ at $0{ }^{\circ} \mathrm{C}$. $\mathrm{AlMe}_{3}(2.0 \mathrm{M}$ in toluene, $9.0 \mathrm{~mL}, 18.0 \mathrm{mmol}, 2.0$ equiv) was then slowly added. The mixture was stirred at $0{ }^{\circ} \mathrm{C}$ for 30 min and was warmed to room temperature for $30 \mathrm{~min}$. The clear solution was cooled to $0{ }^{\circ} \mathrm{C}$ and a solution of $8(2.67 \mathrm{~g}, 9.0 \mathrm{mmol})$ in $\mathrm{CH}_{2} \mathrm{Cl}_{2}(10 \mathrm{~mL})$ was slowly added. The mixture was allowed to warm to room temperature and was stirred for $1 \mathrm{~h}$. The mixture was then cooled to 0 ${ }^{\circ} \mathrm{C}$ and was quenched by slowly addition of aqueous $\mathrm{HCl}$ solution $(1.0 \mathrm{M}, 18 \mathrm{~mL})$; gentle bubbling was observed. The aqueous layers were extracted with $\mathrm{CH}_{2} \mathrm{Cl}_{2}(2 \times 20 \mathrm{~mL})$. The combined organic layers were dried $\left(\mathrm{Na}_{2} \mathrm{SO}_{4}\right)$, and filtered. The solvent was removed by rotary evaporation to give a crude product, which was purified by chromatography (silica gel, hexane/EtOAc, 4/1 to 3/7) to afford $2.99 \mathrm{~g}(93 \%)$ of $\mathbf{1 5}$ as a pale yellow oil. (This reaction can be run on a $40.0 \mathrm{mmol}$ scale in $88 \%$ yield.)

Analytical Data for 15:

1․

$6.51\left(\mathrm{dd}, J=7.0,1.0,1 \mathrm{H}, \mathrm{HC}\left(1^{\prime}\right)\right), 6.14$ (t, $\left.J=8.0,1 \mathrm{H}, \mathrm{HC}\left(2^{\prime}\right)\right), 4.49$ (br dd, $J=$ 7.2, 4.8, $1 \mathrm{H}, \mathrm{HC}(2)), 4.06(\mathrm{dt}, J=8.0,7.0,1 \mathrm{H}, \mathrm{HC}(4)), 3.79-3.73$ (m, $2 \mathrm{H}$, $\left.\mathrm{H}_{2} \mathrm{C}(4)\right), 3.72$ (s, $3 \mathrm{H}, \mathrm{H}_{3} \mathrm{C}\left(2^{\prime}\right.$ ') ), 3.22 (s, $3 \mathrm{H}, \mathrm{H}_{3} \mathrm{C}\left(1^{\prime}\right.$ ')), 2.38 (br s, $\left.1 \mathrm{H}, \mathrm{HO}\right)$ ), 1.98-1.87 (m, $2 \mathrm{H}, \mathrm{H}_{2} \mathrm{C}(3)$ ), 1.76 (d quint, $J=14.0,7.5,1 \mathrm{H}, \mathrm{HC}\left(4^{\prime}\right)$ ), 1.58 (d quint, $\left.J=14.5,7.5,1 \mathrm{H}, \mathrm{HC}\left(4^{\prime}\right)\right), 0.97$ (t, $J=7.5,3 \mathrm{H}, \mathrm{H}_{3} \mathrm{C}\left(5^{\prime}\right)$ )

${ }^{13} \underline{\mathrm{C} \mathrm{NMR}}: \quad\left(126 \mathrm{MHz}, \mathrm{CDCl}_{3}\right)$

$172.8(\mathrm{C}(1)), 141.5\left(\mathrm{C}\left(2^{\prime}\right)\right), 84.9$ (C(1’)), $81.5\left(\mathrm{C}\left(3^{\prime}\right)\right), 72.6(\mathrm{C}(2)), 61.5\left(\mathrm{C}\left(2^{\prime}\right.\right.$ ’) )), $60.0(\mathrm{C}(4)), 34.5(\mathrm{C}(3)), 32.4\left(\mathrm{C}\left(1^{\prime \prime}\right)\right), 27.6\left(\mathrm{C}\left(4^{\prime}\right)\right), 9.1\left(\mathrm{C}\left(5^{\prime}\right)\right)$ 
IR: $\quad(\mathrm{NaCl})$

3436 (s), 2964 (m), 2935 (s), 2879 (s), 1735 (m), 1658 (s), 1461 (s), 1390 (s), 1330 (s), 1274 (s), 1180 (s), 1103 (s), 995 (s), 943 (m), 717 (s)

MS: $\quad(\mathrm{EI}, 70 \mathrm{eV})$

$358\left(27, \mathrm{M}^{+}+1\right), 340$ (11), 269 (7), 230 (13), 211 (12), 195 (100), 164 (49), 146 (38), 141 (35), 116 (14), 88 (10), 75 (33), 68 (61), 61 (45)

Opt. Rot.: $\quad[\alpha]_{\mathrm{D}}^{24}+67.5\left(c=1.08, \mathrm{CHCl}_{3}\right)$

TLC: $\quad R_{f} 0.14$ (silica gel, EtOAc/hexane, 7/3, PMA)

Analysis: $\quad \mathrm{C}_{11} \mathrm{H}_{20} \mathrm{O}_{4} \mathrm{NI} \quad(357.19)$

$\begin{array}{lllll}\text { Calculated: } & \text { C, 36.99; } & \text { H, 5.64, } & \text { N, 3.92, } & \text { I, 35.53\% } \\ \text { Found: } & \text { C, 36.84; } & \text { H, 5.56, } & \text { N, 3.86, } & \text { I, 35.55\% }\end{array}$

\section{Preparation of (2S)- $N$-Methoxy- $N$-methyl-2-\{[(1S,2Z)-1-ethyl-3-iodo-2-propenyl]oxy $\}-4-(4-$ methoxybenzyloxy)butanamide (9)}
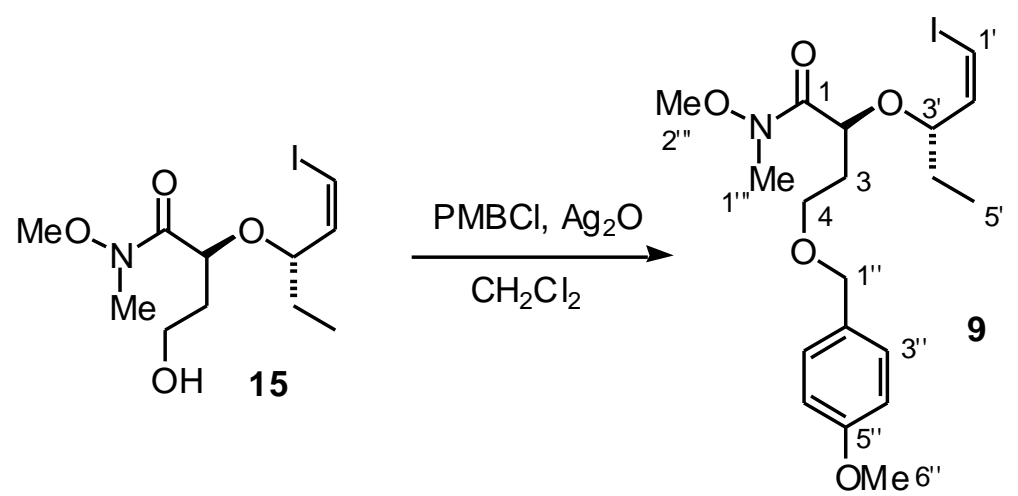

In a three-neck, $50 \mathrm{~mL}$ flask was placed $15(7.14 \mathrm{~g}, 20 \mathrm{mmol})$ in $\mathrm{CH}_{2} \mathrm{Cl}_{2}(20 \mathrm{~mL})$ under $\mathrm{N}_{2}$ at room temperature. $p$-Methoxybenzyl chloride $(4.7 \mathrm{~g}, 30 \mathrm{mmol}, 1.5$ equiv) and freshly prepared $\mathrm{Ag}_{2} \mathrm{O}$ (2.78 g, $12 \mathrm{mmol}, 0.6$ equiv) was then added sequentially. The mixture was stirred for $6 \mathrm{~h}$ and additional $p$-methoxybenzyl chloride ( $4.7 \mathrm{~g}, 30 \mathrm{mmol}, 1.5$ equiv) and $\mathrm{Ag}_{2} \mathrm{O}$ (2.78 g, $12 \mathrm{mmol}, 0.6$ equiv) was added sequentially. After $6 \mathrm{~h}$ stirring, additional $p$ methoxybenzyl chloride (3.13 g, $20 \mathrm{mmol}, 1.0$ equiv) and $\mathrm{Ag}_{2} \mathrm{O}$ (2.78 g, $12 \mathrm{mmol}, 0.6$ equiv) was added again and the mixture was stirred for $12 \mathrm{~h}$. The mixture was filtered through short column of Celite, which was then eluted with $\mathrm{Et}_{2} \mathrm{O}(500 \mathrm{~mL})$. The eluate was concentrated by rotary evaporation to give a crude product, which was purified by chromatography (silica gel, hexane/Et $2 \mathrm{O}, 9 / 1$ to $7 / 3$, then hexane/EtOAc, $1 / 1$ to $3 / 7)$ to afford $7.82 \mathrm{~g}(82 \%)$ of 9 as a colorless oil and $0.57 \mathrm{~g}(8 \%)$ of $\mathbf{1 5}$. (This reaction can be run on a $60 \mathrm{mmol}$ scale in $80 \%$ yield.) 
Analytical Data for 9:

${ }^{1}$ H NMR: $\quad\left(500 \mathrm{MHz}, \mathrm{CDCl}_{3}\right)$

$7.24\left(\mathrm{dd}, J=8.5,1.5,2 \mathrm{H}, 2 \times \mathrm{HC}\left(3^{\prime}{ }^{\prime}\right)\right), 6.86\left(\mathrm{dd}, J=8.5,1.5,2 \mathrm{H} .2 \times \mathrm{HC}\left(4^{\prime}\right)\right)$ ), $6.40\left(\mathrm{dd}, J=7.5,1.0,1 \mathrm{H}, \mathrm{HC}\left(1^{\prime}\right)\right), 6.03$ (t, $\left.J=8.0,1 \mathrm{H}, \mathrm{HC}\left(2^{\prime}\right)\right), 4.45$ (d, $J=$ 12.0, $1 \mathrm{H}, \mathrm{HC}\left(1^{\prime}\right.$ ') ), 4.43-4.41 (m, $\left.1 \mathrm{H}, \mathrm{HC}(2)\right), 4.36$ (d, $J=12.0,1 \mathrm{H}, \mathrm{HC}\left(1^{\prime}\right.$ ')), 4.02 (dt, $\left.J=8.0,6.5,1 \mathrm{H}, \mathrm{HC}\left(3^{\prime}\right)\right), 3.79$ (s, $3 \mathrm{H}, \mathrm{H}_{3} \mathrm{C}\left(6^{\prime}\right.$ ')), 3.68 (s, $3 \mathrm{H}$, $\mathrm{H}_{3} \mathrm{C}(2$ ', $)$ ), 3.63 (td, $\left.J=9.0,5.0,1 \mathrm{H}, \mathrm{HC}(4)\right), 3.53$ (ddd, $J=9.5,5.5,4.5,1 \mathrm{H}$,

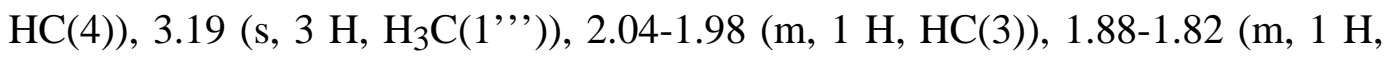
$\mathrm{HC}(3)$ ), 1.74 (d quint, $J=14.0,7.5,1 \mathrm{H}, \mathrm{HC}\left(4^{\prime}\right)$ ), 1.58 (d quint, $J=14.5,7.5,1$ $\left.\mathrm{H}, \mathrm{HC}\left(4^{\prime}\right)\right), 0.95$ (t, $\left.J=7.5,3 \mathrm{H}, \mathrm{H}_{3} \mathrm{C}\left(5^{\prime}\right)\right)$

${ }^{13}$ C NMR: $\quad\left(126 \mathrm{MHz}, \mathrm{CDCl}_{3}\right)$

173.4 (C(1)), 159.0 (C(5’’)), 141.9 (C(2’)), 130.5 (C(2’’)), 129.0 (2 x C(3’’)), 113.5 (2 x C(4’)), 84.0 (C(1')), 81.3 (C(3’)), 72.2 (C(2)), 70.6 (C(1'’)), 65.8 (C(4)), $61.3\left(\mathrm{C}\left(2^{\prime \prime \prime}\right)\right), 55.1\left(\mathrm{C}\left(6^{\prime \prime}\right)\right), 32.5\left(\mathrm{C}(3), \mathrm{C}\left(1^{\prime \prime},\right)\right), 27.4\left(\mathrm{C}\left(4^{\prime}\right)\right), 9.0\left(\mathrm{C}\left(5^{\prime}\right)\right)$

IR: $\quad(\mathrm{NaCl})$

2964 (s), 2935 (s), 2873 (s), 1735 (m), 1675 (s), 1612 (s), 1513 (s), 1461 (s), 1247 (s), 1174 (s), 1099 (s), 1035 (s), 997 (s), 821 (m), 715 (m)

MS: (FAB)

$478\left(20, \mathrm{M}^{+}+1\right), 448$ (3), 352 (5), 284 (2), 195 (3), 146 (9), 121 (100)

Opt. Rot.: $\quad[\alpha]_{\mathrm{D}}{ }^{24}+80.7\left(c=1.20, \mathrm{CHCl}_{3}\right)$

TLC: $\quad R_{f} 0.19$ (silica gel, hexane/EtOAc, 3/2, PMA)

Analysis: $\quad \mathrm{C}_{19} \mathrm{H}_{28} \mathrm{O}_{5} \mathrm{NI} \quad(477.34)$

Calculated: $\quad$ C, 47.79; H, 5.91, N, 2.94, I, 26.59\%

Found: $\quad$ C, 48.12; H, 5.90, N, 2.97, I, 26.79\%


Preparation of

methoxybenzyloxy)butanal (4)
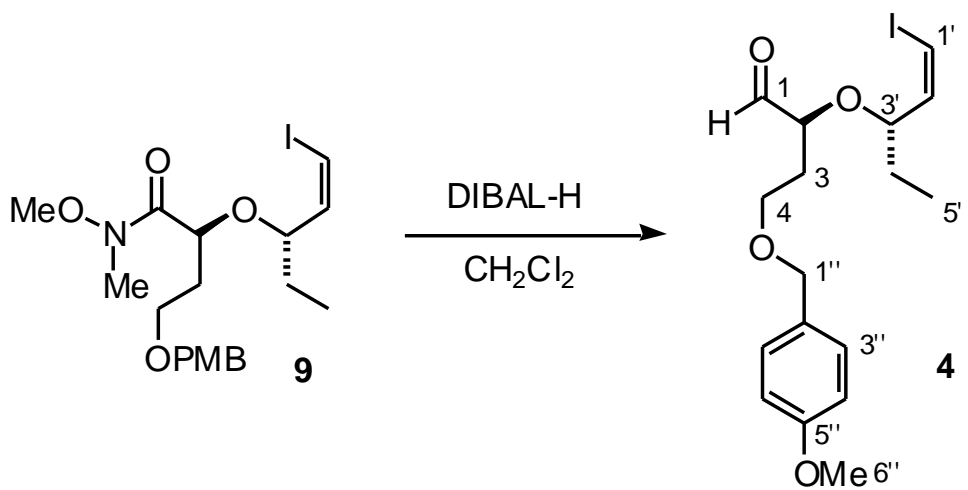

In a three-neck, $100 \mathrm{~mL}$ flask was placed 9 (7.16 g, $15 \mathrm{mmol})$ in $\mathrm{CH}_{2} \mathrm{Cl}_{2}(30 \mathrm{~mL})$ under $\mathrm{N}_{2}$ at $-73{ }^{\circ} \mathrm{C}$. A solution of DIBAL-H (1.0 M in hexane, $22.5 \mathrm{~mL}, 22.5 \mathrm{mmol}, 1.5$ equiv) was then slowly added. The mixture was stirred for $3 \mathrm{~h}$ at $-73^{\circ} \mathrm{C}$ and acetone $(5.0 \mathrm{~mL})$ was added dropwise. The mixture was stirred for $1 \mathrm{~h}$ at $-73{ }^{\circ} \mathrm{C}$. The mixture was then poured into a vigorously stirring mixture of $\mathrm{CH}_{2} \mathrm{Cl}_{2}(50 \mathrm{~mL}) /$ aqueous $\mathrm{HCl}$ solution $(3.0 \mathrm{M}, 50 \mathrm{~mL})$ and was then separated. The aqueous layer was extracted with $\mathrm{CH}_{2} \mathrm{Cl}_{2}(3 \times 50 \mathrm{~mL})$. The combined organic layers were dried $\left(\mathrm{Na}_{2} \mathrm{SO}_{4}\right)$, and filtered. The solvent was removed by rotary evaporation to give a crude product, which was purified by chromatography (silica gel, hexane/EtOAc, 9/1 to 17/3) to afford $5.45 \mathrm{~g}(87 \%)$ of 4 as a pale yellow oil. (This reaction can be run on a $25.0 \mathrm{mmol}$ scale in $88 \%$ yield.)

\section{Analytical Data for 4:}

1 MR: $\quad\left(500 \mathrm{MHz}, \mathrm{CDCl}_{3}\right)$

$9.70(\mathrm{~d}, J=1.5,1 \mathrm{H}, \mathrm{HC}(1)), 7.23$ (dd, $J=8.5,2.0,2 \mathrm{H}, 2$ x HC(3’')), 6.87 (dd, $J$ $=8.5,2.0,2$ H. 2 x HC(4'’)), $6.44\left(\mathrm{dd}, J=8.0,1.0,1 \mathrm{H}, \mathrm{HC}\left(1^{\prime}\right)\right), 6.01(\mathrm{t}, J=8.0$, $\left.1 \mathrm{H}, \mathrm{HC}\left(2^{\prime}\right)\right), 4.41$ (d, $J=11.0,1 \mathrm{H}, \mathrm{HC}\left(1^{\prime}\right.$ ') $), 4.38$ (d, $J=11.0,1 \mathrm{H}, \mathrm{HC}\left(1^{\prime}\right.$ ')), $4.13\left(\mathrm{dt}, J=8.0,6.0,1 \mathrm{H}, \mathrm{HC}\left(3^{\prime}\right)\right), 3.96(\mathrm{ddd}, J=7.5,4.5,1.5,1 \mathrm{H}, \mathrm{HC}(2)), 3.80$ (s, $3 \mathrm{H}, \mathrm{H}_{3} \mathrm{C}\left(6^{\prime}\right.$ ') ), 3.57 (ddd, $\left.J=9.5,6.0,5.0,1 \mathrm{H}, \mathrm{HC}(4)\right), 3.52$ (ddd, $J=9.5$, 7.5, 6.0, $1 \mathrm{H}, \mathrm{HC}(4)$ ), 1.98 (ddt, $J=14.5,7.5,5.0,1 \mathrm{H}, \mathrm{HC}(3)$ ), 1.86 (dddd, $J=$ 14.0, 7.5, 6.0, 5.0, $1 \mathrm{H}, \mathrm{HC}(3)), 1.74$ (d quint, $J=14.5,7.5,1 \mathrm{H}, \mathrm{HC}\left(4^{\prime}\right)$ ), 1.59 (d quint, $\left.J=14.0,7.5,1 \mathrm{H}, \mathrm{HC}\left(4^{\prime}\right)\right), 0.99$ (t, $\left.J=7.5,3 \mathrm{H}, \mathrm{H}_{3} \mathrm{C}\left(5^{\prime}\right)\right)$ 
${ }^{13}$ C NMR: $\quad\left(126 \mathrm{MHz}, \mathrm{CDCl}_{3}\right)$

203.2 (C(1)), 159.1 (C(5')), 140.8 (C(2')), 130.2 (C(2’')), 129.2 (2 x C(3’')),

113.7 (2 x C(4’')), 85.1 (C(1')), 82.2 (C(3')), 78.5 (C(2)), 75.2 (C(1',)), 64.9 (C(4)), 55.2 (C(6’')), $31.1(\mathrm{C}(3)), 27.5\left(\mathrm{C}\left(4^{\prime}\right)\right), 9.4\left(\mathrm{C}\left(5^{\prime}\right)\right)$

IR: $\quad(\mathrm{NaCl})$

2962 (m), 2933 (m), 2873 (m), 1731 (s), 1612 (m), 1513 (s), 1461 (m), 1301 (m), 1247 (s), 1174 (m), 1101 (s), 1035 (s), 821 (m), 715 (m)

MS: (FD)

$418\left(100, \mathrm{M}^{+}\right), 389$ (1), 290 (7), 202 (1)

Opt. Rot.: $\quad[\alpha]_{\mathrm{D}}{ }^{24}+34.7\left(c=1.19, \mathrm{CHCl}_{3}\right)$

TLC: $\quad R_{f} 0.30$ (silica gel, hexane/EtOAc, 4/1, PMA)

Analysis: $\quad \mathrm{C}_{17} \mathrm{H}_{23} \mathrm{O}_{4} \mathrm{I} \quad$ (418.27)

Calculated: $\quad$ C, 48.82; $\quad$ H, 5.54, I, 30.34\%

Found: $\quad$ C, $49.04 ; \quad H, 5.60, \quad$ I, 30.56\%

Preparation of $\quad(4 R, 5 S)-5-\{[(1 S, 2 Z)-1-E t h y l-3-i o d o-2-p r o p e n y l] o x y\}-7-(4-$ methoxybenzyloxy)-1-hepten-4-ol (10)

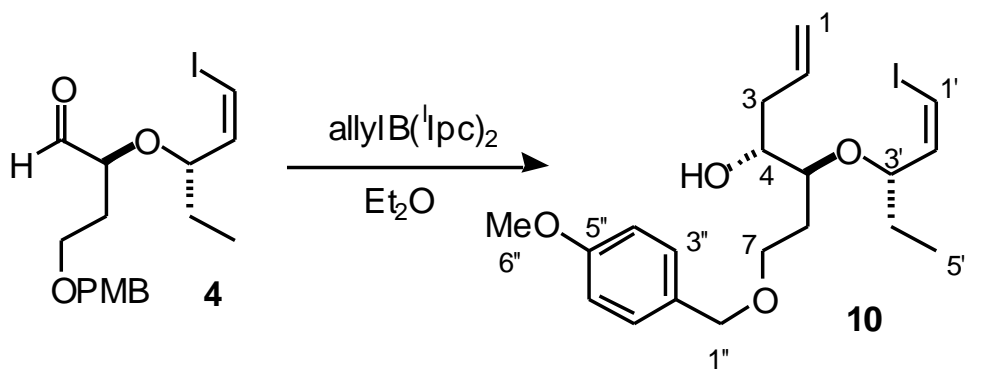

In a three-neck, $100 \mathrm{~mL}$ flask was placed (+)- $B$-chlorodiisopinocampheylborane $[(+)$ DIP-Cl] (4.81 g, $15 \mathrm{mmol}, 1.5$ equiv, purchased from Aldrich) in $\mathrm{Et}_{2} \mathrm{O}$ (15 mL) under $\mathrm{N}_{2}$ at -73 ${ }^{\circ} \mathrm{C}$. Allylmagnesium bromide $\left(0.75 \mathrm{M}\right.$ in $\mathrm{Et}_{2} \mathrm{O}, 20 \mathrm{~mL}, 15 \mathrm{mmol}, 1.5$ equiv) was then added dropwise. After complete addition of Grignard reagent, the mixture was allowed to warm to room temperature and was stirred for $1 \mathrm{~h}$. The solvent was then removed in vacuo. The residue was extracted with pentane $(20 \mathrm{~mL})$ and was stirred vigorously for $30 \mathrm{~min}$. The mixture was then filtered to a receiver flask by cannula under $\mathrm{N}_{2}$. The white salt was washed with pentane (3 $\mathrm{x} 10 \mathrm{~mL}$ ) and was then filtered. The filtrate was concentrated in vacuo to give corresponding allylborane reagent as a colorless oil. To the crude reagent was added $\mathrm{Et}_{2} \mathrm{O}(30 \mathrm{~mL})$ and was then cooled to -95 to $-100{ }^{\circ} \mathrm{C}(\mathrm{EtOH} /$ liquid nitrogen bath). A solution of 4 (4.18 g, $10 \mathrm{mmol})$ in 
$\mathrm{Et}_{2} \mathrm{O}(10 \mathrm{~mL})$ was added among the surface of flask over 10 min under $\mathrm{N}_{2}$. The flask was rinsed with $\mathrm{Et}_{2} \mathrm{O}(5 \mathrm{~mL})$ and was then added to the reaction flask. The mixture was stirred at -95 to $-100{ }^{\circ} \mathrm{C}$ for $2 \mathrm{~h}$ and was concentrated in vacuo to afford crude product. To the crude product was added pentane $(10 \mathrm{~mL})$ and ethanolamine $(1.81 \mathrm{~mL}, 30 \mathrm{mmol}, 3.0$ equiv) sequentially under $\mathrm{N}_{2}$ at room temperature. The mixture was stirred for $12 \mathrm{~h}$ and was then filtered through a short column of Celite, which was eluted with pentane $(300 \mathrm{~mL})$. The solvent was removed by rotary evaporation to give crude product, which was purified by chromatography (silica gel, hexane/EtOAc, 19/1 to 17/3) to afford $4.10 \mathrm{~g}(89 \%)$ of $\mathbf{1 0}$ as a colorless oil. (This reaction can be run on a $20.0 \mathrm{mmol}$ scale $85 \%$ yield.)

\section{Analytical Data for 10:}

1․

$7.24\left(\mathrm{dd}, J=9.0,2.0,2 \mathrm{H}, 2 \times \mathrm{HC}\left(3^{\prime \prime}\right)\right), 6.88(\mathrm{dd}, J=8.5,2.0,2$ H. 2 × $\mathrm{HC}(4$ '’)), 6.34 (d, $\left.J=8.0,1 \mathrm{H}, \mathrm{HC}\left(1^{\prime}\right)\right), 6.01$ (t, $\left.J=8.0,1 \mathrm{H}, \mathrm{HC}\left(2^{\prime}\right)\right), 5.88$ (ddt, $J=17.0$, 10.0, 6.5, $1 \mathrm{H}, \mathrm{HC}(2)), 5.14$ (dt, $J=17.0,1.5,1 \mathrm{H}, \mathrm{HC}(1)), 5.10$ (dd, $J=10.0,1.0$, $1 \mathrm{H}, \mathrm{HC}(1)), 4.43$ (d, $J=11.5,1 \mathrm{H}, \mathrm{HC}(1 '),), 4.39$ (d, $J=11.5,1 \mathrm{H}, \mathrm{HC}(1 ')$,$) ,$ 4.14 (dd, $\left.J=8.0,6.5,1 \mathrm{H}, \mathrm{HC}\left(3^{\prime}\right)\right), 3.91-3.87$ (m, $\left.1 \mathrm{H}, \mathrm{HC}(4)\right), 3.80$ (s, $3 \mathrm{H}$, $\mathrm{H}_{3} \mathrm{C}(6$ ') ), 3.57 (dt, $J=9.5,5.5,1 \mathrm{H}, \mathrm{HC}(7))$, 3.52-3.46 (m, $\left.2 \mathrm{H}, \mathrm{HC}(7), \mathrm{HC}(5)\right)$, 2.51 (br s, $1 \mathrm{H}, \mathrm{HO}), 2.25$ (t, $\left.J=7.0,2 \mathrm{H}, \mathrm{H}_{2} \mathrm{C}(3)\right)$, 1.83-1.74 (m, $\left.2 \mathrm{H}, \mathrm{H}_{2} \mathrm{C}(6)\right)$, 1.64 (d quint, $J=14.0,7.5,1 \mathrm{H}, \mathrm{HC}\left(4^{\prime}\right)$ ), 1.52 (d quint, $J=14.0,7.5,1 \mathrm{H}$, $\left.\mathrm{HC}\left(4^{\prime}\right)\right), 0.95$ (t, $\left.J=7.5,3 \mathrm{H}, \mathrm{H}_{3} \mathrm{C}\left(5^{\prime}\right)\right)$

${ }^{13}$ C NMR: $\quad\left(126 \mathrm{MHz}, \mathrm{CDCl}_{3}\right)$

159.2 (C(5')), 141.9 (C(2’)), 135.1 (C(2)), 130.4 (C(2’')), 129.3 (2 x C(3’’)), 117.3 (C(1)), 113.7 (2 x C(4’’)), 83.8 (C(1')), 80.9 (C(3’)), 76.5 (C(5)), 72.6 $\left(\mathrm{C}\left(1^{\prime \prime}\right)\right), 70.8(\mathrm{C}(4)), 66.2(\mathrm{C}(7)), 55.2\left(\mathrm{C}\left(6^{\prime \prime}\right)\right), 37.1(\mathrm{C}(3)), 29.0(\mathrm{C}(6)), 27.6$ $\left(\mathrm{C}\left(4^{\prime}\right)\right), 9.5\left(\mathrm{C}\left(5^{\prime}\right)\right)$

IR: $\quad(\mathrm{NaCl})$

3467 (m), 3072 (m), 2962 (s), 2933 (s), 2873 (s), 1641 (m), 1612 (s), 1513 (s), 1461 (m), 1247 (s), 1174 (m), 1085 (s), 1035 (s), 916 (m), 821 (m), 713 (m)

MS: $\quad(\mathrm{FD})$

$460\left(100, \mathrm{M}^{+}\right), 425$ (2), 390 (1), 332 (16), 306 (3), 264 (3), 195 (2)

Opt. Rot.: $\quad[\alpha]_{\mathrm{D}}^{24}+58.0\left(c=1.21, \mathrm{CHCl}_{3}\right)$

TLC: $\quad R_{f} 0.15$ (silica gel, hexane/EtOAc, 4/1, PMA)

Analysis: $\quad \mathrm{C}_{20} \mathrm{H}_{29} \mathrm{O}_{4} \mathrm{I} \quad(460.37)$

Calculated: $\quad$ C, 52.18; $\quad$ H, 6.35\%

Found: $\quad$ C, $52.52 ; \quad$ H, $6.37 \%$ 
Preparation of Dimethyl-\{(4R,5S)-5-\{[(1S,2Z)-1-ethyl-3-iodo-2-propenyl]oxy $\}-7-(4-$ methoxybenzyloxy)-1-hepten-4-oxy\}vinylsilane (16)

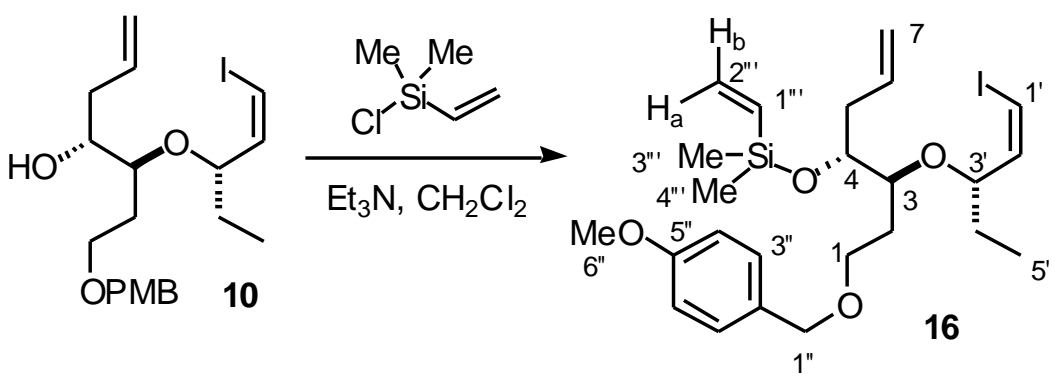

In a three-neck, $50 \mathrm{~mL}$ flask was placed $\mathbf{1 0}(2.3 \mathrm{~g}, 5.0 \mathrm{mmol})$ in $\mathrm{CH}_{2} \mathrm{Cl}_{2}(10 \mathrm{~mL})$ under $\mathrm{N}_{2}$ atmosphere at $0{ }^{\circ} \mathrm{C}$. $\mathrm{Et}_{3} \mathrm{~N}(1.04 \mathrm{~mL}, 7.5 \mathrm{mmol}, 1.5$ equiv) and dimethylvinylchlorosilane ( $0.83 \mathrm{~mL}, 6.0 \mathrm{mmol}, 1.2$ equiv) was then added sequentially. The white suspension was allowed to warm to room temperature and was stirred for $30 \mathrm{~min}$. The mixture was poured into ice water $(10 \mathrm{~mL})$ and the aqueous layer was extracted with $\mathrm{CH}_{2} \mathrm{Cl}_{2}(2 \times 10 \mathrm{~mL})$. The combined organic layers were dried $\left(\mathrm{Na}_{2} \mathrm{SO}_{4}\right)$, and filtered. The solvent was removed by rotary evaporation to give crude product, which was purified by chromatography (silica gel, hexane/EtOAc, 19/1) followed by Kugelrohr distillation to afford $2.48 \mathrm{~g}(91 \%)$ of $\mathbf{1 6}$ as a colorless oil. (This reaction can be run on a $20.0 \mathrm{mmol}$ scale $90 \%$ yield.)

Analytical Data for 16:

bp: $\quad 210-215^{\circ} \mathrm{C}(0.05 \mathrm{mmHg}, \mathrm{ABT})$

${ }^{1} \underline{\mathrm{H} \mathrm{NMR}}: \quad\left(500 \mathrm{MHz}, \mathrm{CDCl}_{3}\right)$

$7.25(\mathrm{dd}, J=9.0,2.0,2 \mathrm{H}, 2$ x HC(3'”)), 6.87 (dd, $J=8.5,2.0,2$ H. 2 x HC(4’)), $6.32\left(\mathrm{dd}, J=7.5,1.0,1 \mathrm{H}, \mathrm{HC}\left(1^{\prime}\right)\right), 6.18\left(\mathrm{dd}, J=20.5,15.0,1 \mathrm{H}, \mathrm{HC}\left(1^{\prime}{ }^{\prime \prime}\right)\right)$ ), 6.00 (t, $\left.J=8.0,1 \mathrm{H}, \mathrm{HC}\left(2^{\prime}\right)\right), 5.99$ (dd, $J=15.0,4.0,1 \mathrm{H}, \mathrm{H}_{\mathrm{b}} \mathrm{C}\left(2^{\prime}\right.$ ', $)$ ), 5.83 (ddt, $J=$ 17.0, 10.0, 7.0, $1 \mathrm{H}, \mathrm{HC}(6)), 5.76\left(\mathrm{dd}, J=20.5,4.0,1 \mathrm{H}, \mathrm{H}_{\mathrm{a}} \mathrm{C}(2\right.$ '”' )), 5.08-5.03 $\left(\mathrm{m}, 2 \mathrm{H}, \mathrm{H}_{2} \mathrm{C}(7)\right), 4.43\left(\mathrm{~d}, J=11.5,1 \mathrm{H}, \mathrm{HC}\left(1 '{ }^{\prime}\right)\right), 4.39(\mathrm{~d}, J=11.0,1 \mathrm{H}$, $\mathrm{HC}\left(1^{\prime}\right.$ ') ), 4.20 (q, $\left.J=7.5,1 \mathrm{H}, \mathrm{HC}\left(3^{\prime}\right)\right), 3.86$ (ddd, $\left.J=7.5,5.0,2.5,1 \mathrm{H}, \mathrm{HC}(4)\right)$, 3.80 (s, $\left.3 \mathrm{H}, \mathrm{H}_{3} \mathrm{C}\left(6^{\prime \prime}\right)\right)$ ), 3.52 (dd, $\left.J=6.5,2.0,1 \mathrm{H}, \mathrm{HC}(1)\right), 3.50$ (dd, $J=6.5,4.5$, $1 \mathrm{H}, \mathrm{HC}(1)), 3.44$ (ddd, $J=7.5,4.0,2.5,1 \mathrm{H}, \mathrm{HC}(3))$, 2.29-2.16 (m, $2 \mathrm{H}$, $\mathrm{H}_{2} \mathrm{C}(5)$ ), 1.77-1.64 (m, $3 \mathrm{H}, \mathrm{H}_{2} \mathrm{C}(2), \mathrm{HC}\left(4^{\prime}\right)$ ), 1.53 (d quint, $J=15.0,7.5,1 \mathrm{H}$, $\left.\mathrm{HC}\left(4^{\prime}\right)\right), 0.93$ (t, $\left.J=7.5,3 \mathrm{H}, \mathrm{H}_{3} \mathrm{C}\left(5^{\prime}\right)\right), 0.21$ (s, $3 \mathrm{H}, \mathrm{H}_{3} \mathrm{C}\left(3^{\prime},{ }^{\prime}\right)$ ), 0.20 (s, $3 \mathrm{H}$, $\left.\mathrm{H}_{3} \mathrm{C}(4,, ')\right)$ 
${ }^{13}$ C NMR: $\quad\left(126 \mathrm{MHz}, \mathrm{CDCl}_{3}\right)$

159.0 (C(5’)), $142.4\left(\mathrm{C}\left(2^{\prime}\right)\right), 138.3$ (C(1'’’)), 135.7 (C(6)), 132.8 (C(2’’')), 130.7

$\left(\mathrm{C}\left(2^{\prime \prime}\right)\right), 129.2$ (2 x C(3’’)), 116.7 (C(7)), 113.6 (2 x C(4’')), 83.4 (C(1')), 81.1

(C(3’)), 76.6 (C(3)), $73.8(\mathrm{C}(4)), 72.4\left(\mathrm{C}\left(1^{\prime \prime}\right)\right), 66.8(\mathrm{C}(1)), 55.2\left(\mathrm{C}\left(6^{\prime \prime}\right)\right), 38.2$

(C(5)), $30.2(\mathrm{C}(2)), 27.7\left(\mathrm{C}\left(4^{\prime}\right)\right), 9.3\left(\mathrm{C}\left(5^{\prime}\right)\right),-1.1\left(\mathrm{C}\left(3^{\prime}, '\right)\right),-1.4\left(\mathrm{C}\left(4^{\prime \prime},\right)\right)$

IR: $\quad(\mathrm{NaCl})$

2962 (s), 2875 (m), 1612 (m), 1513 (m), 1463 (m), 1249 (s), 1097 (s), 1037 (s),

$914(\mathrm{~m}), 833$ (s), $784(\mathrm{~s}), 701$ (m)

MS: (FD)

$544\left(100, \mathrm{M}^{+}\right), 454$ (1), 416 (9), 348 (1), 290 (1), 224 (2), 195 (2), 155 (10), 121

(9)

Opt. Rot.: $\quad[\alpha]_{\mathrm{D}}^{24}+39.9\left(c=1.12, \mathrm{CHCl}_{3}\right)$

TLC: $\quad R_{f} 0.29$ (silica gel, hexane/EtOAc, 9/1, PMA)

Analysis: $\quad \mathrm{C}_{24} \mathrm{H}_{37} \mathrm{O}_{4} \mathrm{SiI} \quad(544.55)$

Calculated: C, 52.94; H, 6.85, I, 23.30\%

Found: $\quad$ C, 53.33; $\quad$ H, 7.00, I, 23.37\%

Preparation of $(6 R)-2,2-D i m e t h y l-6-\{(1 S)-1-\{[(1 S, 2 Z)-1-e t h y l-3-i o d o-2-p r o p e n y l] o x y\}-3-(4-$ methoxybenzyloxy)propan-1-yl\}-1-oxa-2-silacyclohex-3-ene (3)

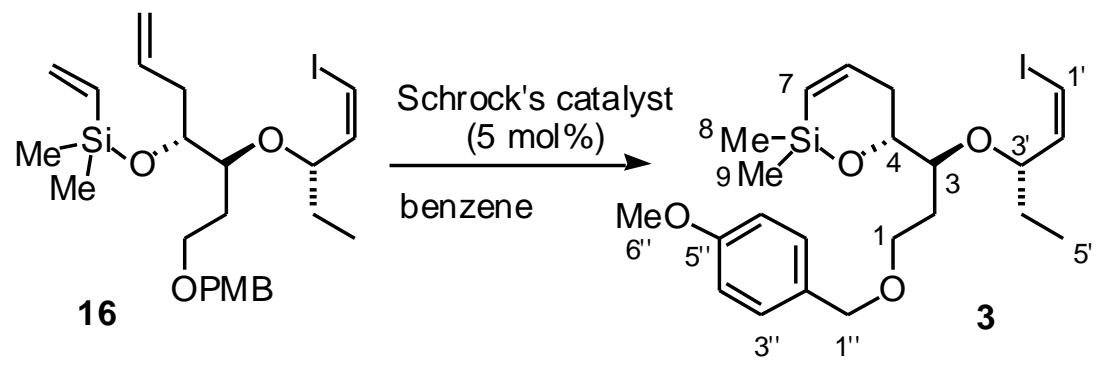

In a flame-dried, $25 \mathrm{ml}$ flask was placed freshly distilled benzene $(15 \mathrm{~mL})$, which was then moved into a dry box. To this flask was added 16 (816 mg, $1.5 \mathrm{mmol})$ and Schrock's catalyst $^{3}$ (57.4 mg, $0.075 \mathrm{mmol}, 0.05$ equiv) sequentially. The yellow-brown solution was stirred at room temperature for $1 \mathrm{~h}$ in the dry box. The solvent was removed by rotary evaporation to give a brown residue, which was purified by chromatography (silica gel, hexane/EtOAc, 19/1 to 9/1) to afford $713 \mathrm{mg}(92 \%)$ of $\mathbf{3}$ as a pale yellow oil. (This reaction can be run on a $5.0 \mathrm{mmol}$ scale $92 \%$ yield.) 
Analytical Data for 3:

${ }^{1}$ H NMR: $\quad\left(500 \mathrm{MHz}, \mathrm{CDCl}_{3}\right)$

$7.26\left(\mathrm{dd}, J=9.0,2.0,2 \mathrm{H}, 2 \times \mathrm{HC}\left(3^{\prime}\right.\right.$ ') $), 6.88$ (dd, $J=9.0,2.0,2$ H. 2 x HC(4'”)), $6.80(\mathrm{ddd}, J=14.0,6.0,2.0,1 \mathrm{H}, \mathrm{HC}(6)), 6.32\left(\mathrm{dd}, J=7.5,0.5,1 \mathrm{H}, \mathrm{HC}\left(1^{\prime}\right)\right)$, $6.03\left(\mathrm{t}, J=7.5,1 \mathrm{H}, \mathrm{HC}\left(2^{\prime}\right)\right), 5.75(\mathrm{dd}, J=14.0,2.5,1 \mathrm{H}, \mathrm{HC}(7)), 4.42$ (s, $2 \mathrm{H}$, $\left.\mathrm{H}_{2} \mathrm{C}\left(1^{\prime},\right)\right), 4.30$ (dt, $\left.J=8.0,6.5,1 \mathrm{H}, \mathrm{HC}\left(3^{\prime}\right)\right), 4.03$ (dt, $J=11.5,3.5,1 \mathrm{H}$, $\mathrm{HC}(4)), 3.80$ (s, $3 \mathrm{H}, \mathrm{H}_{3} \mathrm{C}\left(6^{\prime}\right.$ ')), 3.53 (t, $\left.J=6.5,2 \mathrm{H}, \mathrm{H}_{2} \mathrm{C}(1)\right), 3.49$ (dt, $J=8.0$, 4.0, $1 \mathrm{H}, \mathrm{HC}(3)), 2.30$ (ddt, $J=17.5,11.0,2.5,1 \mathrm{H}, \mathrm{HC}(5)$ ), 2.11 (ddd, $J=17.5$, 6.5, 2.0, $1 \mathrm{H}, \mathrm{HC}(5))$, 1.81-1.74 (m, $2 \mathrm{H}, \mathrm{H}_{2} \mathrm{C}(2)$ ), 1.65 (d quint, $J=14.5,7.5,1$ $\mathrm{H}, \mathrm{HC}\left(4^{\prime}\right)$ ), 1.51 (d quint, $\left.J=14.0,7.5,1 \mathrm{H}, \mathrm{HC}\left(4^{\prime}\right)\right), 0.96$ (t, $J=7.5,3 \mathrm{H}$, $\left.\mathrm{H}_{3} \mathrm{C}\left(5^{\prime}\right)\right), 0.18$ (s, $\left.3 \mathrm{H}, \mathrm{H}_{3} \mathrm{C}(8)\right), 0.17$ (s, $\left.3 \mathrm{H}, \mathrm{H}_{3} \mathrm{C}(9)\right)$

${ }^{13} \mathrm{C} \mathrm{NMR}: \quad\left(126 \mathrm{MHz}, \mathrm{CDCl}_{3}\right)$

$159.1\left(\mathrm{C}\left(5^{\prime \prime}\right)\right), 147.1(\mathrm{C}(6)), 142.4\left(\mathrm{C}\left(2^{\prime}\right)\right), 130.6\left(\mathrm{C}\left(2^{\prime \prime}\right)\right), 129.2$ (2 x C(3’’)), 127.1 (C(7)), 113.7 (2 x C(4’’)), 83.2 (C(1')), 81.7 (C(3’)), 76.9 (C(3)), 72.7 (C(4)), $72.5\left(\mathrm{C}\left(1^{\prime \prime}\right)\right), 66.7(\mathrm{C}(1)), 55.2\left(\mathrm{C}\left(6^{\prime \prime}\right)\right), 31.7(\mathrm{C}(5)), 30.6(\mathrm{C}(2)), 27.7$ $\left(\mathrm{C}\left(4^{\prime}\right)\right), 9.7\left(\mathrm{C}\left(5^{\prime}\right)\right),-0.4(\mathrm{C}(8)),-0.7(\mathrm{C}(9))$

IR: $\quad(\mathrm{NaCl})$

2960 (s), 2933 (s), 2858 (s), 1612 (s), 1587 (s), 1513 (s), 1463 (m), 1355 (m), 1249 (s), 1091 (s), 1037 (s), 956 (s), 906 (m), 840 (s), 788 (s), 713 (m)

MS: $\quad(\mathrm{EI}, 70 \mathrm{eV})$

$517\left(3, \mathrm{M}^{+}+1\right), 379(5), 321(5), 303$ (2), 215 (5), 195 (3), 185 (30), 127 (20), $121(100), 68(4)$

Opt. Rot.: $\quad[\alpha]_{\mathrm{D}}^{24}+45.1\left(c=1.02, \mathrm{CHCl}_{3}\right)$

TLC: $\quad R_{f} 0.25$ (silica gel, hexane/EtOAc, 9/1, PMA)

Analysis: $\quad \mathrm{C}_{22} \mathrm{H}_{33} \mathrm{O}_{4} \mathrm{SiI} \quad$ (516.60)

Calculated: $\quad$ C, 51.16; $\quad$ H, 6.44, I, $24.57 \%$

Found: $\quad$ C, 51.21; $\quad$ H, 6.56, I, 24.30\% 
Preparation of $(2 S, 8 R, 9 S)$-2-Ethyl-8-hydroxy-9-[2-(4-methoxybenzyloxy)ethyl]-1-oxa3Z,5Z-cyclononadiene (2)

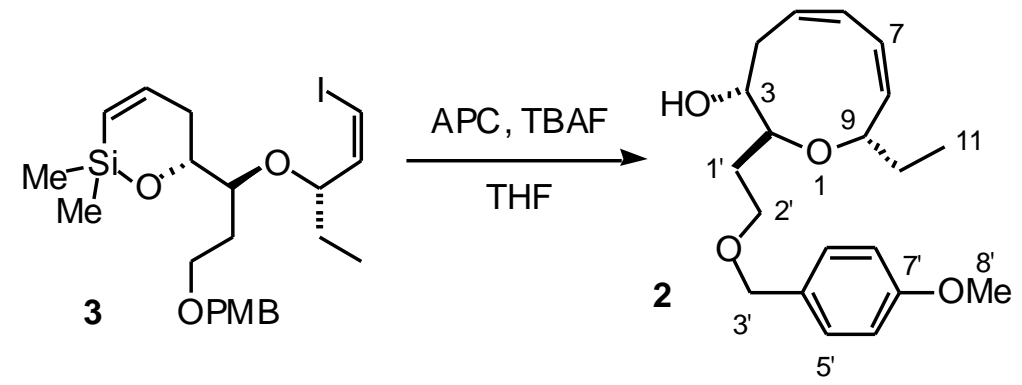

In a three-neck, $50 \mathrm{~mL}$ flask was placed $\mathrm{TBAF} \bullet 3 \mathrm{H}_{2} \mathrm{O}(9.47 \mathrm{~g}, 30.0 \mathrm{mmol}, 10.0$ equiv $)$ in THF (30 mL) under $\mathrm{N}_{2}$ at room temperature. $\pi$-Allylpalladium chloride dimer (APC) (82.4 mg, $0.225 \mathrm{mmol}, 0.075$ equiv, purchased from ACROS) was added. A solution of 3 (1.55 g, 3.0 $\mathrm{mmol})$ in THF (10 mL) was then added by syringe pump for $60 \mathrm{~h}$. After complete addition of $\mathbf{3}$, the mixture was stirred for $1 \mathrm{~h}$ and was then concentrated. The residue was filtered through a short silica gel column, which was eluted with $\mathrm{Et}_{2} \mathrm{O}(600 \mathrm{~mL})$. The solvent was removed by rotary evaporation to give a brown residue, which was purified by chromatography (silica gel, hexane/EtOAc, 9/1 to 4/1) to afford $607 \mathrm{mg}(61 \%)$ of 2 as a colorless oil.

Analytical Data for 2:

${ }^{1}$ H NMR: $\quad\left(500 \mathrm{MHz}, \mathrm{CDCl}_{3}\right)$

$7.23\left(\mathrm{dd}, J=8.5,2.0,2 \mathrm{H}, 2 \times \mathrm{HC}\left(5^{\prime}\right)\right), 6.87\left(\mathrm{dd}, J=8.5,2.0,2 \mathrm{H}, 2 \times \mathrm{HC}\left(6^{\prime}\right)\right)$, 6.16 (ddd, $J=11.0,3.0,1.5,1 \mathrm{H}, \mathrm{HC}(7)), 5.93$ (d, $J=11.0,1 \mathrm{H}, \mathrm{HC}(6)$ ), 5.87$5.82(\mathrm{~m}, 1 \mathrm{H}, \mathrm{HC}(5)), 5.49$ (ddd, $J=11.5,6.0,2.0,1 \mathrm{H}, \mathrm{HC}(8)), 4.48(\mathrm{~d}, J=11.5$, $\left.1 \mathrm{H}, \mathrm{HC}\left(3^{\prime}\right)\right), 4.41$ (d, $\left.J=11.5,1 \mathrm{H}, \mathrm{HC}\left(3^{\prime}\right)\right), 4.04$ (dt, $\left.J=7.0,6.5,1 \mathrm{H}, \mathrm{HC}(9)\right)$, 3.88 (dt, $J=8.0,4.0,1 \mathrm{H}, \mathrm{HC}(2)), 3.80-3.76$ (m, $\left.1 \mathrm{H}, \mathrm{HC}\left(2^{\prime}\right)\right), 3.79$ (s, $3 \mathrm{H}$, $\mathrm{H}_{3} \mathrm{C}\left(8^{\prime}\right)$ ), 3.68-3.55 (br s, $1 \mathrm{H}, \mathrm{HO}$ ), 3.57 (td, $J=8.5,2.0,1 \mathrm{H}, \mathrm{HC}(3)$ ), 3.54 (ddd, $\left.J=9.0,6.0,3.0,1 \mathrm{H}, \mathrm{HC}\left(2^{\prime}\right)\right), 2.61(\mathrm{dt}, J=13.0,9.0,1 \mathrm{H}, \mathrm{HC}(4)), 2.30(\mathrm{dd}, J=$ 13.0, 7.0, $1 \mathrm{H}, \mathrm{HC}(4)), 2.10$ (ddt, $\left.J=15.5,9.0,3.5,1 \mathrm{H}, \mathrm{HC}\left(1^{\prime}\right)\right), 1.83$ (dddd, $J=$ 15.5, 6.0, 4.5, 3.0, $1 \mathrm{H}, \mathrm{HC}\left(1^{\prime}\right)$ ), 1.60 (d quint, $J=14.5,7.5,1 \mathrm{H}, \mathrm{HC}(10)$ ), 1.47 (d quint, $J=14.5,7.5,1 \mathrm{H}, \mathrm{HC}(10)), 0.85$ (t, $J=7.5,3 \mathrm{H}, \mathrm{H}_{3} \mathrm{C}(11)$ )

${ }^{13} \mathrm{C} \mathrm{NMR}: \quad\left(126 \mathrm{MHz}, \mathrm{CDCl}_{3}\right)$

159.3 (C(7’), 134.1 (C(8)), 133.0 (C(7)), 130.6 (C(5)), $129.7\left(\mathrm{C}\left(4^{\prime}\right)\right), 129.4$ (2 x C(5’)), 127.1 (C(6)), 113.8 (2 x C(6’)), 78.8 (C(2)), 75.0 (C(9)), 73.0 (C(3’)), 70.6 (C(3)), 66.0 (C(2')), 55.2 (C(8')), 36.9 (C(4)), 33.8 (C(1')), 29.1 (C(10)), 10.2 $(\mathrm{C}(11))$ 
IR: $\quad(\mathrm{NaCl})$

3419 (s), 3004 (s), 2960 (s), 2931 (s), 2873 (s), 1612 (s), 1513 (s), 1463 (s), 1363

(m), 1301 (s), 1247 (s), 1174 (s), 1064 (s), 1039 (s), 821 (m), 754 (s)

MS: (FD)

$$
332\left(100, \mathrm{M}^{+}\right), 166(1), 121(2)
$$

Opt. Rot.: $\quad[\alpha]_{\mathrm{D}}^{24}+162.7\left(c=0.9, \mathrm{CHCl}_{3}\right)$

TLC: $\quad R_{f} 0.18$ (silica gel, hexane/EtOAc, 7/3, PMA)

Analysis: $\quad \mathrm{C}_{20} \mathrm{H}_{28} \mathrm{O}_{4} \quad(332.44)$
Calculated:
C, 72.26;
$\mathrm{H}, 8.49 \%$
C, 71.99;
$\mathrm{H}, 8.35 \%$

Found:

Preparation of $\quad(2 S, 8 R, 9 S)-8-(t-B u t y l d i m e t h y l s i l y l o x y)-2-e t h y l-9-[2-(4-$ methoxybenzyloxy)ethyl]-1-oxa-3Z,5Z-cyclononadiene (17)

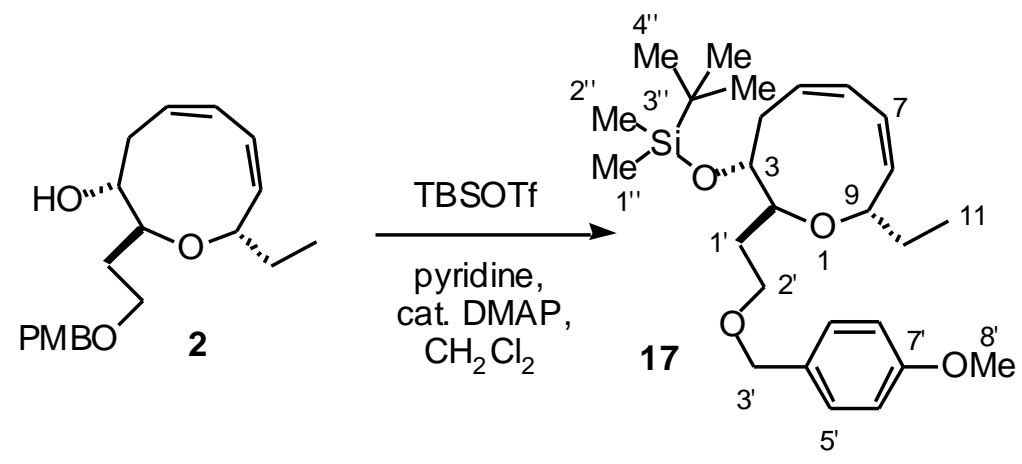

In a three-neck, $50 \mathrm{~mL}$ flask was placed $2(1.99 \mathrm{~g}, 6.0 \mathrm{mmol})$ in $\mathrm{CH}_{2} \mathrm{Cl}_{2}(12 \mathrm{~mL})$ under $\mathrm{N}_{2}$ at $0{ }^{\circ} \mathrm{C}$. Pyridine (1.45 mL, $18 \mathrm{mmol}, 3.0$ equiv) and 4-dimethylaminopyridine (73.2 $\mathrm{mg}, 0.6$ mmol, 0.1 equiv) was added sequentially. To the mixture was then added TBSOTf $(2.07 \mathrm{~mL}, 9.0$ mmol, 1.5 equiv) dropwise. The mixture was allowed to warm to room temperature and was stirred for $2 \mathrm{~h}$. The mixture was poured into $\mathrm{H}_{2} \mathrm{O}(12 \mathrm{~mL})$ and the aqueous layer was extracted with $\mathrm{CH}_{2} \mathrm{Cl}_{2}$ ( $\left.3 \times 12 \mathrm{~mL}\right)$. The combined organic layers were dried $\left(\mathrm{Na}_{2} \mathrm{SO}_{4}\right)$ and filtered. The solvent was removed by rotary evaporation to give crude product, which was purified by chromatography (silica gel, hexane/EtOAc, 49/1 to 19/1) to afford $2.36 \mathrm{~g}(88 \%)$ of 17 as a colorless oil. 
Analytical Data for 17:

bp: $\quad 195-200{ }^{\circ} \mathrm{C}(0.3 \mathrm{mmHg}, \mathrm{ABT})$

1․

$7.28\left(\mathrm{dd}, J=8.5,2.0,2 \mathrm{H}, 2 \times \mathrm{HC}\left(5^{\prime}\right)\right), 6.89\left(\mathrm{dd}, J=8.5,2.0,2 \mathrm{H}, 2 \times \mathrm{HC}\left(6^{\prime}\right)\right)$, $6.00(\mathrm{dd}, J=11.0,1.0,1 \mathrm{H}, \mathrm{HC}(7)), 5.88-5.82$ (m, $2 \mathrm{H}, \mathrm{HC}(5), \mathrm{HC}(6)), 5.51$ (dd, $J=11.5,7.5,1 \mathrm{H}, \mathrm{HC}(8)), 4.47\left(\mathrm{~d}, J=11.5,1 \mathrm{H}, \mathrm{HC}\left(3^{\prime}\right)\right), 4.43(\mathrm{~d}, J=11.5,1 \mathrm{H}$, HC(3')), $4.06(\mathrm{td}, J=8.0,4.5,1 \mathrm{H}, \mathrm{HC}(9)), 3.83$ (td, $J=8.5,3.0,1 \mathrm{H}, \mathrm{HC}(2))$, 3.82 (s, $\left.3 \mathrm{H}, \mathrm{H}_{3} \mathrm{C}\left(8^{\prime}\right)\right)$ ) 3.73 (ddd, $\left.J=8.5,6.5,2.0,1 \mathrm{H}, \mathrm{HC}(3)\right), 3.63(\mathrm{td}, J=9.0$, 5.0, $\left.1 \mathrm{H}, \mathrm{HC}\left(2^{\prime}\right)\right), 3.58$ (td, $\left.J=9.0,7.0,1 \mathrm{H}, \mathrm{HC}\left(2^{\prime}\right)\right)$, 2.95 (ddd, $J=15.5,8.5$, 7.0, $1 \mathrm{H}, \mathrm{HC}(4)$ ), 2.20-2.16 (m, $1 \mathrm{H}, \mathrm{HC}(4)$ ), 2.04 (dddd, $J=13.5,10.0$, 7.0, 3.0, $\left.1 \mathrm{H}, \mathrm{HC}\left(1^{\prime}\right)\right), 1.84$ (dtd, $\left.J=13.5,8.5,5.0,1 \mathrm{H}, \mathrm{HC}\left(1^{\prime}\right)\right)$, 1.68-1.60 (m, $1 \mathrm{H}$, $\mathrm{HC}(10)$ ), 1.50 (d quint, $J=15.0,7.5,1 \mathrm{H}, \mathrm{HC}(10)$ ), 0.91 (s, $9 \mathrm{H}, 3$ x $\mathrm{H}_{3} \mathrm{C}\left(4^{\prime}\right.$ ')), $0.83\left(\mathrm{t}, J=7.5,3 \mathrm{H}, \mathrm{H}_{3} \mathrm{C}(11)\right), 0.12$ (s, $3 \mathrm{H}, \mathrm{H}_{3} \mathrm{C}\left(1\right.$ '’)), 0.09 (s, $3 \mathrm{H}, \mathrm{H}_{3} \mathrm{C}\left(2^{\prime}\right.$ ') )

${ }^{13}$ C NMR: $\quad\left(126 \mathrm{MHz}, \mathrm{CDCl}_{3}\right)$

$159.1\left(\mathrm{C}\left(7^{\prime}\right), 133.9(\mathrm{C}(8)), 130.8\left(\mathrm{C}\left(4^{\prime}\right)\right), 130.40(\mathrm{C}(5)), 130.37(\mathrm{C}(7)), 129.2(2 \mathrm{x}\right.$ $\left.\mathrm{C}\left(5^{\prime}\right)\right), 127.1$ (C(6)), 113.7 (2 x C(6’)), 81.8 (C(2)), 73.1 (C(3)), 73.0 (C(9)), 72.5 $\left(\mathrm{C}\left(3^{\prime}\right)\right), 67.3\left(\mathrm{C}\left(2^{\prime}\right)\right), 55.2\left(\mathrm{C}\left(8^{\prime}\right)\right), 36.8(\mathrm{C}(4)), 33.3$ (C(1')), $29.4(\mathrm{C}(10)), 25.8$ (3 x C(4'’)), $17.9\left(\mathrm{C}\left(3^{\prime \prime}\right)\right), 9.6(\mathrm{C}(11)),-4.2\left(\mathrm{C}\left(1^{\prime \prime}\right)\right),-4.8\left(\mathrm{C}\left(2^{\prime} ’\right)\right)$

IR: $\quad(\mathrm{NaCl})$

3004 (m), 2956 (s), 2931 (s), 2858 (s), 1614 (m), 1513 (s), 1463 (m), 1361 (m), 1301 (m), 1249 (s), 1172 (m), 1085 (s), 1039 (s), 1006 (m), 943 (m), 836 (s), 775 (s)

MS: (FD)

$446\left(100, \mathrm{M}^{+}\right), 389$ (3), 332 (6), 215 (2), 121 (3)

Opt. Rot.: $\quad[\alpha]_{\mathrm{D}}{ }^{24}+74.9\left(c=1.155, \mathrm{CHCl}_{3}\right)$

TLC: $\quad R_{f} 0.29$ (silica gel, hexane/EtOAc, 9/1, PMA)

Analysis: $\quad \mathrm{C}_{26} \mathrm{H}_{42} \mathrm{O}_{4} \mathrm{Si} \quad$ (446.71)

Calculated: $\quad$ C, 69.91; H, $9.48 \%$

Found: $\quad$ C, $69.85 ; \quad H, 9.50 \%$ 
Preparation of $(2 S, 8 R, 9 S)-8-(t$-Butyldimethylsilyloxy)-2-ethyl-9-(2-hydroxyethyl)-1-oxa3Z,5Z-cyclononadiene (18)

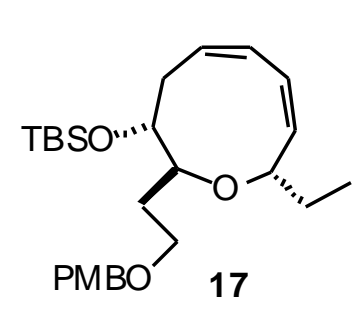

17

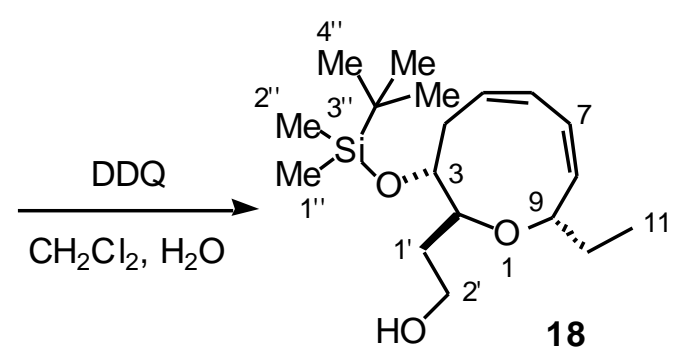

18

In a three-neck, $100 \mathrm{~mL}$ flask was placed $17(2.23 \mathrm{~g}, 5.0 \mathrm{mmol})$ in $\mathrm{CH}_{2} \mathrm{Cl}_{2}(47.5 \mathrm{~mL})$ and $\mathrm{H}_{2} \mathrm{O}(2.5 \mathrm{~mL})$ at room temperature. 2,3-Dichloro-5,6-dicyano-1,4-benzoquinone (DDQ) (1.7 g, $7.5 \mathrm{mmol}, 1.5$ equiv) was then added in one portion. The dark green solution was stirred for 30 min with brown suspension was observed. The mixture was poured into a saturated $\mathrm{NaHCO}_{3}$ solution $(50 \mathrm{~mL})$. The aqueous layer was extracted with $\mathrm{CH}_{2} \mathrm{Cl}_{2}(3 \times 50 \mathrm{~mL})$. The combined organic layers were dried $\left(\mathrm{Na}_{2} \mathrm{SO}_{4}\right)$ and filtered. The solvent was removed by rotary evaporation to give crude product, which was purified by chromatography (silica gel, hexane/Et $2 \mathrm{O}, 9 / 1$, then hexane/EtOAc, 17/3) to afford $1.37 \mathrm{~g}(84 \%)$ of $\mathbf{1 8}$ as a colorless oil.

Analytical Data for 18:

${ }^{1} \underline{\mathrm{H} \mathrm{NMR}}: \quad\left(500 \mathrm{MHz}, \mathrm{CDCl}_{3}\right)$

$6.05(\mathrm{dd}, J=11.0,2.5,1 \mathrm{H}, \mathrm{HC}(7)), 5.86(\mathrm{dd}, J=11.0,2.5,1 \mathrm{H}, \mathrm{HC}(6)), 5.78$ (dt, $J=11.5,8.0,1 \mathrm{H}, \mathrm{HC}(5)), 5.45(\mathrm{dd}, J=11.5,8.0,1 \mathrm{H}, \mathrm{HC}(8)), 4.09(\mathrm{td}, J=8.0$, 6.0, $1 \mathrm{H}, \mathrm{HC}(9)), 3.95(\mathrm{td}, J=8.0,3.0,1 \mathrm{H}, \mathrm{HC}(2)), 3.79(\mathrm{ddd}, J=12.0,7.0,4.5$, $\left.1 \mathrm{H}, \mathrm{HC}\left(2^{\prime}\right)\right), 3.72$ (ddd, $\left.J=12.0,6.5,5.5,1 \mathrm{H}, \mathrm{HC}\left(2^{\prime}\right)\right), 3.68(\mathrm{td}, J=7.5,1.5,1$ H, HC(3)), 2.73 (ddd, $J=14.0,9.5,7.5,1 \mathrm{H}, \mathrm{HC}(4)$ ), 2.66 (br s, $1 \mathrm{H}, \mathrm{HO}$ ), 2.22 (dd, $J=14.0,7.5,1 \mathrm{H}, \mathrm{HC}(4)), 1.90$ (dddd, $J=14.5,6.5,5.0,3.5,1 \mathrm{H}, \mathrm{HC}\left(1^{\prime}\right)$ ), 1.79 (dddd, $J=14.5,8.0,6.5,5.0,1$ H, HC(1')), 1.73-1.65 (m, 1 H, HC(10)), 1.52 (d quint, $J=13.5,7.5,1 \mathrm{H}, \mathrm{HC}(10)), 0.86$ (s, $9 \mathrm{H}, 3 \times \mathrm{H}_{3} \mathrm{C}\left(4^{\prime}\right.$ ')), 0.83 (t, $J=7.5$, $\left.3 \mathrm{H}, \mathrm{H}_{3} \mathrm{C}(11)\right), 0.09$ (s, $3 \mathrm{H}, \mathrm{H}_{3} \mathrm{C}\left(1\right.$ '’)), 0.06 (s, $3 \mathrm{H}, \mathrm{H}_{3} \mathrm{C}(2$ '’))

\section{${ }^{13} \mathrm{C} \mathrm{NMR}: \quad\left(126 \mathrm{MHz}, \mathrm{CDCl}_{3}\right)$}

$133.1(\mathrm{C}(8)), 131.8(\mathrm{C}(7)), 129.7(\mathrm{C}(5)), 127.5(\mathrm{C}(6)), 83.1(\mathrm{C}(2)), 74.0(\mathrm{C}(9))$, 72.6 (C(3)), $61.4\left(\mathrm{C}\left(2^{\prime}\right)\right), 38.0$ (C(4)), 34.7 (C(1')), 29.2 (C(10)), 25.8 (3 x $\left.\mathrm{C}\left(4^{\prime \prime}\right)\right), 17.9\left(\mathrm{C}\left(3^{\prime \prime}\right)\right), 9.8(\mathrm{C}(11)),-4.4\left(\mathrm{C}\left(1^{\prime \prime}\right)\right),-4.9\left(\mathrm{C}\left(2^{\prime \prime}\right)\right)$

IR: $\quad(\mathrm{NaCl})$

3436 (m), 3006 (m), 2956 (s), 2931 (s), 2885 (s), 2858 (s), 1436 (m), 1361 (m), 1255 (s), 1081 (s), 943 (m), 836 (s), 775 (s) 
MS: (FD)

$326\left(100, \mathrm{M}^{+}\right), 267$ (15), $222(2), 116$ (1)

Opt. Rot.: $\quad[\alpha]_{\mathrm{D}}^{24}+103.4\left(c=1.075, \mathrm{CHCl}_{3}\right)$

TLC: $\quad R_{f} 0.17$ (silica gel, hexane/EtOAc, 4/1, PMA)

Analysis: $\quad \mathrm{C}_{18} \mathrm{H}_{34} \mathrm{O}_{3} \mathrm{Si} \quad$ (326.56)

Calculated: $\mathrm{C}, 66.21 ; \quad \mathrm{H}, 10.49 \%$

Found: $\quad$ C, 66.21; H, $10.40 \%$

Preparation of (2 $S, 8 R, 9 S)-8$-( $t$-Butyldimethylsilyloxy)-2-ethyl-9-(2-oxoethyl)-1-oxa-3Z,5Zcyclononadiene (11)
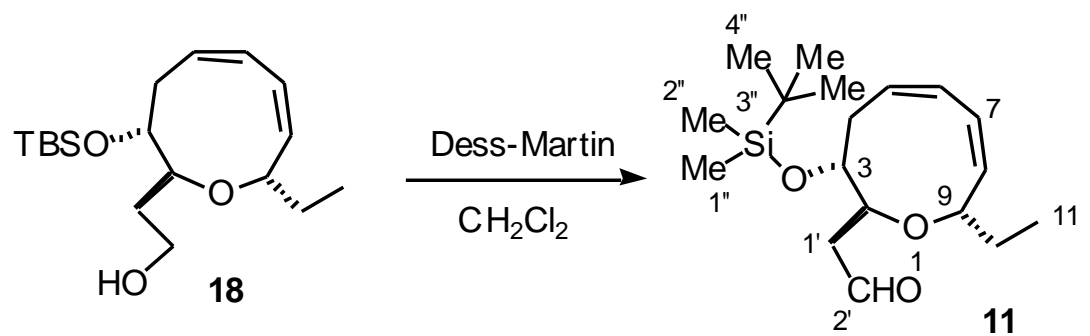

In a three-neck, $50 \mathrm{~mL}$ flask was placed $18(1.14 \mathrm{~g}, 3.5 \mathrm{mmol})$ in $\mathrm{CH}_{2} \mathrm{Cl}_{2}(15 \mathrm{~mL})$ at room temperature. Dess-Martin periodinane (1.93 g, $4.55 \mathrm{mmol}, 1.3$ equiv) was then added in one portion The mixture was stirred for $2 \mathrm{~h}$ and was then filtered through a short silica gel column, which was eluted with hexane/EtOAc $(1 / 1,250 \mathrm{~mL})$. The filtrate was concentrated by rotary evaporation to give crude product, which was purified by chromatography (silica gel, hexane/EtOAc, 19/1) to afford $941 \mathrm{mg}(83 \%)$ of $\mathbf{1 1}$ as a colorless oil.

\section{Analytical Data for 11:}

${ }^{1}$ H NMR: $\quad\left(500 \mathrm{MHz}, \mathrm{CDCl}_{3}\right)$

$9.73\left(\mathrm{t}, J=2.5,1 \mathrm{H}, \mathrm{HC}\left(2^{\prime}\right)\right), 6.10(\mathrm{ddd}, J=11.0,3.0,1.0,1 \mathrm{H}, \mathrm{HC}(7)), 5.89$ (dd, $J=11.0,3.0,1 \mathrm{H}, \mathrm{HC}(6)), 5.78(\mathrm{dt}, J=11.0,8.5,1 \mathrm{H}, \mathrm{HC}(5)), 5.48(\mathrm{ddd}, J=$ 11.0, 7.5, 1.0, $1 \mathrm{H}, \mathrm{HC}(8)), 4.33(\mathrm{td}, J=7.5,5.5,1 \mathrm{H}, \mathrm{HC}(2)), 4.05(\mathrm{q}, J=7.0,1$ H, HC(9)), 3.76 (td, $J=7.5,1.5,1 \mathrm{H}, \mathrm{HC}(3)), 2.73$ (dt, $J=13.5,8.5,1 \mathrm{H}, \mathrm{HC}(4))$, 2.69 (ddd, $J=16.5,5.5,2.0,1 \mathrm{H}, \mathrm{HC}\left(1^{\prime}\right)$ ), 2.56 (ddd, $J=16.0,7.0,3.0,1 \mathrm{H}$, $\left.\mathrm{HC}\left(1^{\prime}\right)\right), 2.22(\mathrm{dd}, J=13.5,7.5,1 \mathrm{H}, \mathrm{HC}(4)), 1.59$ (d quint, $J=14.5,7.5,1 \mathrm{H}$, $\mathrm{HC}(10)$ ), 1.48 (d quint, $J=14.5,7.5,1 \mathrm{H}, \mathrm{HC}(10)$ ), 0.85 (s, $9 \mathrm{H}, 3$ x H $\mathrm{H}_{3} \mathrm{C}\left(4^{\prime}\right.$ ')), $0.82\left(\mathrm{t}, J=7.5,3 \mathrm{H}, \mathrm{H}_{3} \mathrm{C}(11)\right), 0.09$ (s, $3 \mathrm{H}, \mathrm{H}_{3} \mathrm{C}\left(1^{\prime}\right.$ ') ), 0.04 (s, $3 \mathrm{H}, \mathrm{H}_{3} \mathrm{C}\left(2^{\prime}\right.$ ') ) 
${ }^{13}$ C NMR: $\quad\left(126 \mathrm{MHz}, \mathrm{CDCl}_{3}\right)$

$201.6\left(\mathrm{C}\left(2^{\prime}\right)\right), 133.4(\mathrm{C}(8)), 132.3(\mathrm{C}(7)), 129.4(\mathrm{C}(5)), 127.7$ (C(6)), 78.3 (C(2)),

74.3 (C(9)), 72.8 (C(3)), 48.1 (C(1')), 37.9 (C(4)), 28.8 (C(10)), 25.7 (3 x C(4'’)),

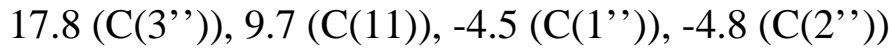

IR: $\quad(\mathrm{NaCl})$

3006 (m), 2956 (s), 2931 (s), 2885 (s), 2858 (s), 2721 (m), 1727 (s), 1463 (m), 1407 (m), 1361 (m), 1257 (s), 1085 (s), 941 (m), 838 (s), 777 (s)

MS: (FD)

$324\left(46, \mathrm{M}^{+}\right), 311$ (3), 299 (11), 283 (83), 271 (16), 267 (31), 211 (3), 199 (2), $183(5), 162(5), 128(3)$

Opt. Rot.: $\quad[\alpha]_{\mathrm{D}}^{24}+113.5\left(c=1.055, \mathrm{CHCl}_{3}\right)$

TLC: $\quad R_{f} 0.36$ (silica gel, hexane/EtOAc, 9/1, PMA)

Analysis: $\quad \mathrm{C}_{18} \mathrm{H}_{32} \mathrm{O}_{3} \mathrm{Si} \quad(324.54)$

Calculated: $\quad$ C, $66.62 ; \quad$ H, $9.95 \%$

Found: $\quad$ C, $65.90 ; \quad$ H, $9.96 \%$

Preparation of $\quad(2 S, 8 R, 9 S)-8$-( $t$-Butyldimethylsilyloxy)-2-ethyl-9-[(2Z)-5(triisopropylsilyl)pent-2-en-4-ynyl]-1-oxa-3Z,5Z-cyclononadiene (19)

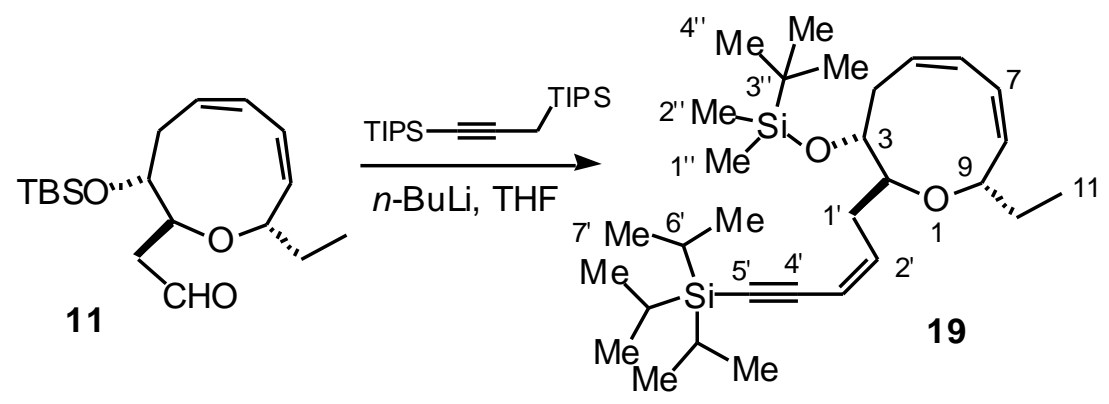

In a three-neck, $25 \mathrm{~mL}$ flask was placed 1,3-bis(triisopropylsilyl)propyne (1.06 g, 3.0 mmol, 1.5 equiv) in THF $(10 \mathrm{~mL})$ under $\mathrm{N}_{2}$ at $-20{ }^{\circ} \mathrm{C} . n$-BuLi $(1.6 \mathrm{M}$ in hexane, $1.88 \mathrm{~mL}, 3.0$ mmol, 1.5 equiv) was then added dropwise. The yellow solution was stirred for $30 \mathrm{~min}$ at -20 ${ }^{\circ} \mathrm{C}$ and was then cooled to $-74{ }^{\circ} \mathrm{C}$. A solution of 11 (648 mg, $\left.2.0 \mathrm{mmol}\right)$ in THF (5 mL) was added dropwise. The mixture was stirred for $2 \mathrm{~h}$ at $-74{ }^{\circ} \mathrm{C}$ and was then slowly warmed to room temperature over $6 \mathrm{~h}$. The reaction was quenched by adding a saturated $\mathrm{NH}_{4} \mathrm{Cl}$ solution $(10 \mathrm{~mL})$ and was then separated. The aqueous layer was extracted with $\mathrm{Et}_{2} \mathrm{O}(3 \times 10 \mathrm{~mL})$. The combined organic layers were dried $\left(\mathrm{Na}_{2} \mathrm{SO}_{4}\right)$ and filtered. The solvent was removed by rotary evaporation to give crude product, which was purified by chromatography (silica gel, 
hexane/EtOAc, $1 / 0$ to 49/1) to afford $833 \mathrm{mg}(83 \%)$ of 19 as a colorless oil with a 6/1 (Z/E) mixtures. Pure $Z$-isomer can be obtained by radial chromatography (silica gel), which was eluted with hexane. The fraction 1 and 2 refer to $E$ - and $Z$-isomer, respectively.

Analytical Data for 19:

1․

6.07 (ddd, $\left.J=11.0,7.0,6.0,1 \mathrm{H}, \mathrm{HC}\left(2^{\prime}\right)\right), 5.99$ (dd, $\left.J=11.5,2.0,1 \mathrm{H}, \mathrm{HC}(7)\right)$, 5.87-5.80 (m, 2 H, HC(5), HC(6)), 5.59 (dt, J = 11.0, 2.0, 1 H, HC(3')), 5.53 (dd, $J=11.5,7.0,1 \mathrm{H}, \mathrm{HC}(8)), 4.03(\mathrm{td}, J=7.0,5.5,1 \mathrm{H}, \mathrm{HC}(9)), 3.89(\mathrm{td}, J=7.5$, 4.5, $1 \mathrm{H}, \mathrm{HC}(2)), 3.78$ (ddd, $J=8.5,7.0,2.0,1 \mathrm{H}, \mathrm{HC}(3)$ ), 2.91 (ddd, $J=14.0$, 8.5, 7.0, $1 \mathrm{H}, \mathrm{HC}(4)), 2.72\left(\mathrm{dtd}, J=16.5,7.5,1.5,1 \mathrm{H}, \mathrm{HC}\left(1^{\prime}\right)\right), 2.66\left(\mathrm{dddd}^{\prime}\right)=$ 16.5, 6.0, 4.5, 2.5, $1 \mathrm{H}, \mathrm{HC}\left(1^{\prime}\right)$ ), 2.17 (ddd, $\left.J=12.5,6.0,1.0,1 \mathrm{H}, \mathrm{HC}(4)\right)$ ) 1.66 (dqd, $J=15.0,7.5,5.0,1 \mathrm{H}, \mathrm{HC}(10)$ ), 1.50 (d quint, $J=15.5,7.5,1 \mathrm{H}, \mathrm{HC}(10)$ ), 1.10-1.08 (br m, $21 \mathrm{H}, 3$ x HC(6'), 6 x H $3 \mathrm{C}\left(7^{\prime}\right)$ ), 0.88 (s, $9 \mathrm{H}, 3 \times \mathrm{H}_{3} \mathrm{C}\left(4^{\prime}\right.$ ')), 0.80 (t, $\left.J=7.5,3 \mathrm{H}, \mathrm{H}_{3} \mathrm{C}(11)\right), 0.10$ ( $\mathrm{s}, 3 \mathrm{H}, \mathrm{H}_{3} \mathrm{C}\left(1^{\prime}\right.$ ') $), 0.06$ (s, $3 \mathrm{H}, \mathrm{H}_{3} \mathrm{C}\left(2^{\prime}\right.$ ' $)$ )

${ }^{13}$ C NMR: $\quad\left(126 \mathrm{MHz}, \mathrm{CDCl}_{3}\right)$

142.6 (C(2’)), 134.1 (C(8)), 130.5 (C(7)), 130.3 (C(5)), 127.1 (C(6)), 110.5 (C(3’)), 104.1 (C(4')), 95.4 (C(5')), 83.4 (C(2)), 73.1 (C(9)), 72.7 (C(3)), 37.0 (C(4)), 34.4 (C(1')), 29.1 (C(10)), 25.8 (3 x C(4'’)), 18.6 (6 x C(7’)), 17.9 $\left(\mathrm{C}\left(3^{\prime \prime}\right)\right), 11.3$ (3 x C(6’)), 9.7 (C(11)), -4.3 (C(1'’)), -4.8 (C(2’’))

IR: $\quad(\mathrm{NaCl})$

3006 (m), 2942 (s), 2863 (s), 2146 (m), 1737 (w), 1637 (w), 1463 (s), 1388 (m), 1255 (m), 1085 (s), 1043 (m), 1004 (m), 883 (m), 836 (s), 775 (s), 744 (m)

MS: (FD)

$502\left(100, \mathrm{M}^{+}\right), 445(11)$

Opt. Rot.: $\quad[\alpha]_{\mathrm{D}}{ }^{24}+92.1\left(c=1.015, \mathrm{CHCl}_{3}\right)$

TLC: $\quad R_{f} 0.17$ (silica gel, hexane/EtOAc, 49/1, PMA)

Analysis: $\quad \mathrm{C}_{30} \mathrm{H}_{54} \mathrm{O}_{2} \mathrm{Si}_{2} \quad(502.93)$

Calculated: $\mathrm{C}, 71.65 ; \quad \mathrm{H}, 10.82 \%$

Found: $\quad$ C, $71.43 ; \quad H, 10.84 \%$ 
Preparation of $(2 S, 8 R, 9 S)-2-E t h y l-8$-hydroxy-9-[(2Z)-pent-2-en-4-ynyl]-1-oxa-3Z,5Zcyclononadiene (12)

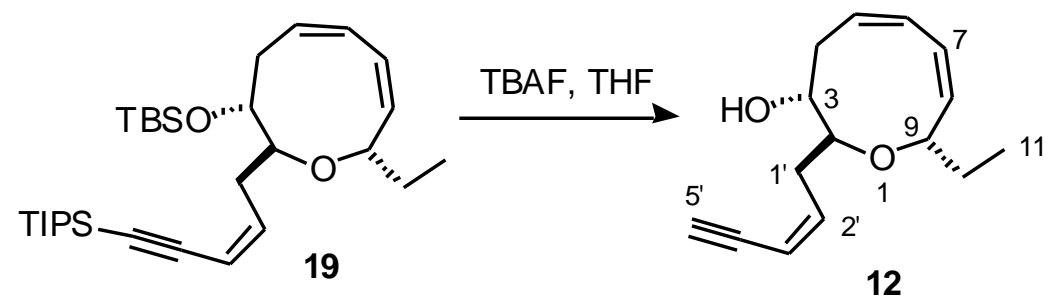

In a three-neck, $50 \mathrm{~mL}$ flask was placed $19(753 \mathrm{mg}, 1.5 \mathrm{mmol})$ in THF $(20 \mathrm{~mL})$ under $\mathrm{N}_{2}$ at $0{ }^{\circ} \mathrm{C}$. A TBAF solution (1.0 M in THF, $6.0 \mathrm{~mL}, 6.0 \mathrm{mmol}, 4.0$ equiv) was then added dropwise. The mixture was stirred $1.5 \mathrm{~h}$ at $0{ }^{\circ} \mathrm{C}$ and was then directly filtered through a short silica gel column, which was eluted with $\mathrm{Et}_{2} \mathrm{O}(500 \mathrm{~mL})$. The solvent was removed by rotary evaporation to give crude product, which was purified by chromatography (silica gel, hexane/EtOAc, 9/1 to 17/3) to afford $324 \mathrm{mg}$ (93\%) of $\mathbf{1 2}$ as a white solid. Compound $\mathbf{1 2}$ can be further recrystallized as needles from pentane at $-30^{\circ} \mathrm{C}$.

Analytical Data for 12:

mp: $\quad 49-50{ }^{\circ} \mathrm{C}$ (pentane)

${ }^{1}$ H NMR: $\quad\left(500 \mathrm{MHz}, \mathrm{CDCl}_{3}\right)$

6.23 (dddd, $\left.J=11.0,9.0,6.0,1.0,1 \mathrm{H}, \mathrm{HC}\left(2^{\prime}\right)\right), 6.12$ (ddd, $J=11.5,2.5,1.5,1 \mathrm{H}$, HC(7)), 5.92 (ddd, $J=11.0,1.5,1.0,1 \mathrm{H}, \mathrm{HC}(6)$ ), 5.80 (ddd, $J=10.5,9.5,7.0,1$ H, HC(5)), 5.58 (d, $\left.J=11.0,1 \mathrm{H}, \mathrm{HC}\left(3^{\prime}\right)\right)$, 5.55 (dd, $J=11.5,6.0,1 \mathrm{H}, \mathrm{HC}(8)$ ), 4.07 (q, $J=7.0,1 \mathrm{H}, \mathrm{HC}(9)$ ), 3.87 (dt, $J=8.5,5.0,1 \mathrm{H}, \mathrm{HC}(2)), 3.60$ (tdd, $J=$ 8.5, 5.0, 2.5, $1 \mathrm{H}, \mathrm{HC}(3)), 3.14\left(\mathrm{~d}, J=2.0,1 \mathrm{H}, \mathrm{HC}\left(5^{\prime}\right)\right), 2.90-2.84(\mathrm{~m}, 1 \mathrm{H}$, $\left.\mathrm{HC}\left(1^{\prime}\right)\right), 2.70(\mathrm{dt}, J=13.0,9.5,1 \mathrm{H}, \mathrm{HC}(4)), 2.58$ (dtd, $J=15.0,5.3,1.5,1 \mathrm{H}$, HC(1')), 2.28 (ddd, $J=13.0,7.0,1.0,1$ H, HC(4)), 2.10 (br s, 1 H, HO)), 1.70 (d quint, $J=15.0,7.5,1 \mathrm{H}, \mathrm{HC}(10)$ ), 1.55 (d quint, $J=15.5,7.5,1 \mathrm{H}, \mathrm{HC}(10)), 0.89$ (t, $J=7.5,3 \mathrm{H}, \mathrm{H}_{3} \mathrm{C}\left(11^{\prime}\right)$ )

${ }^{13}$ C NMR: $\quad\left(126 \mathrm{MHz}, \mathrm{CDCl}_{3}\right)$

$142.5\left(\mathrm{C}\left(2^{\prime}\right)\right), 134.1(\mathrm{C}(8)), 132.6(\mathrm{C}(7)), 130.0(\mathrm{C}(5)), 127.4(\mathrm{C}(6)), 109.8$ $\left(\mathrm{C}\left(3^{\prime}\right)\right), 82.2\left(\mathrm{C}\left(5^{\prime}\right)\right), 80.5\left(\mathrm{C}\left(4^{\prime}\right)\right), 79.7(\mathrm{C}(2)), 74.9(\mathrm{C}(9)), 70.3(\mathrm{C}(3)), 36.5$ $(\mathrm{C}(4)), 34.5\left(\mathrm{C}\left(1^{\prime}\right)\right), 28.9(\mathrm{C}(10)), 10.1(\mathrm{C}(11))$ 
IR: $\quad(\mathrm{NaCl})$

3411 (s, OH), 3298 (s, -CC-H), 3006 (s), 2964 (s), 2931 (s), 2877 (s)), 2096 (w), 1279 (w), 1619 (w), 1440 (s), 1378 (m), 1353 (m), 1240 (m), 1122 (s), 1062 (s), $917(\mathrm{~m}), 892(\mathrm{~m}), 858(\mathrm{~m}), 752(\mathrm{~s})$

MS: $\quad(\mathrm{EI}, 70 \mathrm{eV})$

$233\left(7, \mathrm{M}^{+}+1\right), 232\left(3, \mathrm{M}^{+}\right), 215$ (9), 203 (11), 187 (5), 185 (4), 167 (17), 149 (23), 131 (18), 121 (21), 107 (100), 95 (44), 79 (26), 67 (18)

Opt. Rot.: $\quad[\alpha]_{\mathrm{D}}{ }^{24}+382.5\left(c=1.03, \mathrm{CHCl}_{3}\right)$

TLC: $\quad R_{f} 0.14$ (silica gel, hexane/EtOAc, 4/1, PMA)

Analysis: $\quad \mathrm{C}_{15} \mathrm{H}_{20} \mathrm{O}_{2} \quad$ (232.33)
Calculated:
C, 77.55;
$\mathrm{H}, 8.68 \%$
Found:
C, 77.84;
$\mathrm{H}, 8.72 \%$

Preparation of $\quad(2 S, 8 S, 9 S)-8-C h l o r o-2-e t h y l-9-[(2 Z)-p e n t-2-e n-4-y n y l]-1-o x a-3 Z, 5 Z-$ cyclononadiene $[(+)$-Brasilenyne (1)]

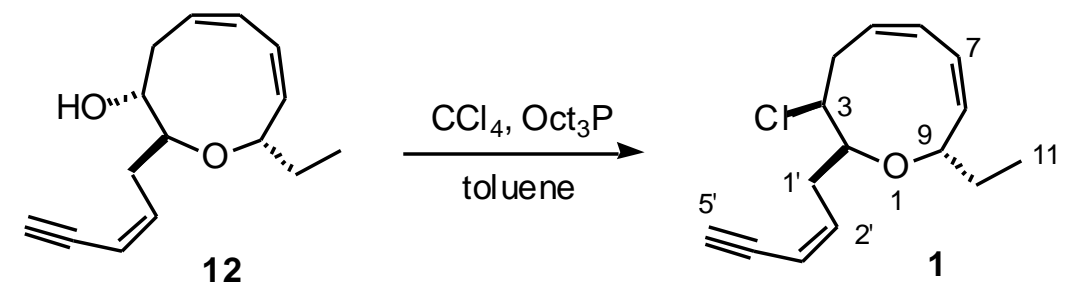

In a three-neck, $25 \mathrm{~mL}$ flask was placed $12(232 \mathrm{mg}, 1.0 \mathrm{mmol})$ in toluene $(10 \mathrm{~mL})$ under argon at room temperature. $\mathrm{CCl}_{4}\left(0.48 \mathrm{~mL}, 5.0 \mathrm{mmol}, 5.0\right.$ equiv) and $(n-\mathrm{Oct})_{3} \mathrm{P}(4.46 \mathrm{~mL}, 10.0 \mathrm{mmol}$, 10.0 equiv) was then added sequentially. The mixture was stirred for $30 \mathrm{~min}$ at room temperature and was then warmed to $60-65{ }^{\circ} \mathrm{C}$ for $12 \mathrm{~h}$. After cooling, a saturated aqueous $\mathrm{Na}_{2} \mathrm{SO}_{3}$ solution $(10 \mathrm{~mL})$ was then added and was vigorously stirred for $1 \mathrm{~h}$. The mixture was separated and the aqueous layer was extracted with $\mathrm{Et}_{2} \mathrm{O}(3 \times 10 \mathrm{~mL})$. The combined organic layers were dried $\left(\mathrm{Na}_{2} \mathrm{SO}_{4}\right)$ and filtered. The solvent was removed by rotary evaporation to give crude product, which was purified by chromatography (silica gel, hexane/Et $2 \mathrm{O}, 1 / 0$ to 49/1) followed by recrystallization from pentane at $-70{ }^{\circ} \mathrm{C}$ to afford $230 \mathrm{mg}$ (92\%, three crops) of $\mathbf{1}$ as a white solid. ${ }^{4}$ 
Analytical Data for 1:

mp: $\quad 37-38{ }^{\circ} \mathrm{C}$ (pentane); lit. $36-37{ }^{\circ} \mathrm{C}$

1․

6.23 (ddd, $J=11.0,2.5,1.5,1 \mathrm{H}, \mathrm{HC}(7)), 6.07$ (br d, $J=11.5,1 \mathrm{H}, \mathrm{HC}(6)), 6.04$ (dddd, $\left.J=11.0,8.0,7.0,1.0,1 \mathrm{H}, \mathrm{HC}\left(2^{\prime}\right)\right), 5.81(\mathrm{dtd}, J=11.0,7.5,1.0,1 \mathrm{H}$, $\mathrm{HC}(5)), 5.55$ (ddd, $J=11.0,7.0,1.0,1 \mathrm{H}, \mathrm{HC}(8)), 5.54(\mathrm{dd}, J=11.0,1.0,1 \mathrm{H}$, HC(3’)), 4.29 (q, $J=7.0,1$ H, HC(9)), 4.08 (br d, $J=8.0,1$ H, HC(3)), 3.90 (ddd, $J=8.0,6.5,1.0,1 \mathrm{H}, \mathrm{HC}(2)), 3.13$ (d, $\left.J=2.0,1 \mathrm{H}, \mathrm{HC}\left(5^{\prime}\right)\right), 2.72$ (dtd, J = 14.0, 8.0, 1.0, $\left.1 \mathrm{H}, \mathrm{HC}\left(1^{\prime}\right)\right), 2.67(\mathrm{dtd}, J=13.5,7.5,1.0,1 \mathrm{H}, \mathrm{HC}(4)), 2.61$ (dddd, $J=$ 14.0, 7.0, 6.5, 1.0, $\left.1 \mathrm{H}, \mathrm{HC}\left(1^{\prime}\right)\right), 2.48$ (dd, $\left.J=13.5,8.0,1 \mathrm{H}, \mathrm{HC}(4)\right), 1.72$ (d quint, $J=14.5,7.5,1 \mathrm{H}, \mathrm{HC}(10)$ ), 1.53 (d quint, $J=14.5,7.5,1 \mathrm{H}, \mathrm{HC}(10)$ ), 0.91 (t, $J=7.5,3 \mathrm{H}, \mathrm{H}_{3} \mathrm{C}(11)$ )

${ }^{13}$ C NMR: $\quad\left(126 \mathrm{MHz}, \mathrm{CDCl}_{3}\right)$

$141.39\left(\mathrm{C}\left(2^{\prime}\right)\right), 134.34(\mathrm{C}(8)), 133.52(\mathrm{C}(7)), 129.65(\mathrm{C}(6)), 128.74(\mathrm{C}(5))$, 110.56 (C(3’)), 82.17 (C(5’)), 80.20 (C(4')), 75.65 (C(2)), 75.54 (C(9)), 63.00 (C(3)), 36.41 (C(1')), 35.98 (C(4)), 28.61 (C(10)), 10.08 (C(11))

IR: $\quad(\mathrm{NaCl})$

3294 (s), 3008 (m), 2964 (s), 2933 (s), 2875 (m), 2096 (w), 1729 (w), 1623 (w), 1461 (m), 1434 (m), 1378 (m), 1351 (m), 1297 (m), 1224 (m), 1120 (s), 1087 (s), 1045 (m), $991(\mathrm{~m}), 894(\mathrm{w}), 755(\mathrm{~m})$

MS: $\quad(\mathrm{EI}, 70 \mathrm{eV})$

$251\left(3, \mathrm{M}^{+}+1\right), 250\left(2, \mathrm{M}^{+}\right), 221\left(12, \mathrm{M}^{+}-\mathrm{Et}\right), 215\left(24, \mathrm{M}^{+}-\mathrm{Cl}\right), 197$ (11), 185 (20), 167 (18), 149 (25), 131 (37), 121 (41), 107 (100), 91 (40), 79 (51), 65 (26)

Opt. Rot.: $\quad[\alpha]_{\mathrm{D}}{ }^{24}+228.0\left(c=1.08, \mathrm{CHCl}_{3}\right)$; lit. $[\alpha]_{\mathrm{D}}{ }^{21}+216\left(c=0.017, \mathrm{CHCl}_{3}\right)$

TLC: $\quad R_{f} 0.14$ (silica gel, hexane/EtOAc, 4/1, PMA)

\section{Preparation of Dicobalt complex (20)}

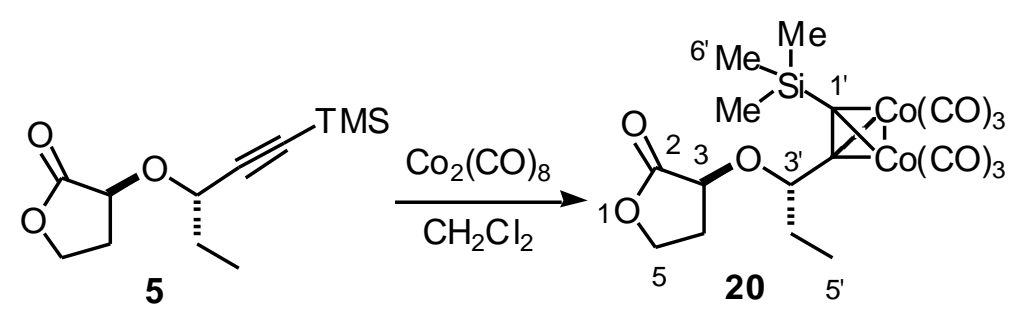

In a three-neck, $25 \mathrm{~mL}$ flask was placed $5(240 \mathrm{mg}, 1.0 \mathrm{mmol})$ in $\mathrm{CH}_{2} \mathrm{Cl}_{2}(5 \mathrm{~mL})$ under $\mathrm{N}_{2}$ at $0{ }^{\circ} \mathrm{C}$. A solution of $\mathrm{Co}_{2}(\mathrm{CO})_{8}\left(684 \mathrm{mg}, 2.0 \mathrm{mmol}, 2.0\right.$ equiv) in $\mathrm{CH}_{2} \mathrm{Cl}_{2}(10 \mathrm{~mL})$ was then 
added. The mixture was allowed to warm to room temperature and was stirred for $2 \mathrm{~h}$. The mixture was directly concentrated to give crude product, which was purified by chromatography (silica gel, hexane/EtOAc, 19/1 to 9/1) to afford $500 \mathrm{mg}(95 \%)$ of $\mathbf{2 0}$ as a red brown solid. (Sublimation of 20 at $65-68{ }^{\circ} \mathrm{C}(0.1 \mathrm{mmHg})$ for overnight gave deep red crystals, which were suitable for X-ray determination.)

Analytical Data for 20:

mp: $\quad 68-69{ }^{\circ} \mathrm{C}$ (sublimation, $0.1 \mathrm{mmHg}$ )

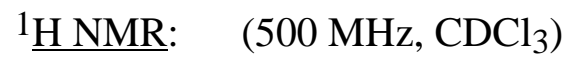

5.03 (dd, $\left.J=7.0,5.5,1 \mathrm{H}, \mathrm{HC}\left(3^{\prime}\right)\right), 4.47$ (dt, $\left.J=9.0,7.0,1 \mathrm{H}, \mathrm{HC}(5)\right), 4.42$ (dd, $J$

$=7.0,5.5,1 \mathrm{H}, \mathrm{HC}(3)), 4.32(\mathrm{td}, J=7.5,5.5,1 \mathrm{H}, \mathrm{HC}(5)), 2.51(\mathrm{dq}, J=14.0,7.0$, $1 \mathrm{H}, \mathrm{HC}(4)), 2.32$ (dq, $J=13.0,5.5,1 \mathrm{H}, \mathrm{HC}(4)), 1.81-1.75$ (m, $2 \mathrm{H}, \mathrm{H}_{2} \mathrm{C}\left(4^{\prime}\right)$ ), 1.08 (t, $\left.J=7.5,3 \mathrm{H}, \mathrm{H}_{3} \mathrm{C}\left(5^{\prime}\right)\right), 0.32$ (s, $9 \mathrm{H}, 3 \times \mathrm{H}_{3} \mathrm{C}\left(7^{\prime}\right)$ )

${ }^{13} \mathrm{C} \mathrm{NMR}: \quad\left(126 \mathrm{MHz}, \mathrm{CDCl}_{3}\right)$

200.3 (6 x C(6')), $175.0(\mathrm{C}(2)), 110.2$ (C(2’)), 80.3 (C(3’)), 78.8 (C(1')), 72.2 $(\mathrm{C}(3)), 66.1(\mathrm{C}(5)), 32.7(\mathrm{C}(4)), 30.0\left(\mathrm{C}\left(4^{\prime}\right)\right), 10.7\left(\mathrm{C}\left(5^{\prime}\right)\right), 1.0\left(\mathrm{C}\left(7^{\prime}\right)\right)$

IR: $\left(\mathrm{CHCl}_{3}\right)$

2967 (s), 2881 (s), 2480 (s), 2086 (s), 2051 (s), 2005 (s), 1770 (s), 1562 (s), 1454

(s), 1380 (s), 1247 (s), 1180 (s), 1099 (s), 1031 (s), 946 (s), 835 (s), 755 (s)

MS: (FAB)

498 (4, M+-CO), 470 (100, M+-2 x CO), 442 (38), 425 (80), 414 (58), 397 (65),

386 (50), 358 (48), 316 (22), 270 (30), 241 (18), 197 (22), 155 (40), 119 (80)

TLC: $\quad R_{f} 0.19$ (silica gel, hexane/EtOAc, 9/1, PMA)

Analysis: $\quad \mathrm{C}_{18} \mathrm{H}_{20} \mathrm{O}_{9} \mathrm{Co}_{2} \mathrm{Si}(526.31)$

Calculated: C, 41.08; H, $3.83 \%$

Found: $\quad$ C, $41.00 ; \quad$ H, $3.64 \%$ 
Table 1. ${ }^{1} \mathrm{H}$ NMR Data of Natural and Synthetic (+)-Brasilenyne (1)

\begin{tabular}{|c|c|c|c|}
\hline $\begin{array}{c}\text { Assignment } \\
\text { (literature) }\end{array}$ & $\begin{array}{c}\text { Natural 1 } \\
\text { Solvent: } \mathrm{CDCl}_{3}(220 \mathrm{MHz})\end{array}$ & $\begin{array}{c}\text { Synthetic 1 } \\
\text { Solvent: } \mathrm{CDCl}_{3}(500 \mathrm{MHz})\end{array}$ & $\begin{array}{c}\text { Assignment } \\
\text { (synthetic) }\end{array}$ \\
\hline $\mathrm{HC}(7)$ & Ref. peak: TMS & $6.23(\mathrm{ddd}, J=11.0,2.5,1.5)$ & $\mathrm{HC}(7)$ \\
$H C\left(3^{\prime}\right)$ & $\sim 6.0(\mathrm{dd}, J=11.0,2.0)$ & $6.07(\mathrm{br} \mathrm{d}, J=11.5)$ & $H C(6)$ \\
$H C(5)$ & $\sim 6.09(\mathrm{~m})$ & $6.04(\mathrm{dddd}, J=11.0,8.0,7.0,1.0)$ & $H C\left(2^{\prime}\right)$ \\
$H C\left(2^{\prime}\right)$ & $5.82(\mathrm{dt}, J=11.0,8.0)$ & $5.81(\mathrm{dtd}, J=11.0,7.5,1.0)$ & $H C(5)$ \\
$\mathrm{HC}(8)$ & $\sim 5.55(\mathrm{dd}, J=10.0,8.0)$ & $5.55(\mathrm{ddd}, J=11.0,7.0,1.0)$ & $\mathrm{HC}(8)$ \\
$H C(6)$ & $\sim 5.55(\mathrm{dd}, J=11.0,2.0)$ & $5.54(\mathrm{dd}, J=11.0,1.0)$ & $H C\left(3^{\prime}\right)$ \\
$\mathrm{HC}(9)$ & $4.29(\mathrm{dtd}, J=8.0,7.0,1.0)$ & $4.29(\mathrm{q}, J=7.0)$ & $\mathrm{HC}(9)$ \\
$H C(2)$ & $4.09(\mathrm{ddd}, J=8.0,3.0,1.0)$ & $4.08(\mathrm{br} \mathrm{d}, J=8.0)$ & $H C(3)$ \\
$H C(3)$ & $3.91(\mathrm{td}, J=7.0,1.0)$ & $3.90(\mathrm{ddd}, J=8.5,6.5,1.0)$ & $H C(2)$ \\
$\mathrm{HC}\left(5^{\prime}\right)$ & $3.14(\mathrm{~d}, J=2.0)$ & $3.13(\mathrm{~d}, J=2.0)$ & $\mathrm{HC}\left(5^{\prime}\right)$ \\
$H C(4)$ & $\sim 2.7(\mathrm{~m})$ & $2.72(\mathrm{dtd}, J=14.0,8.0,1.0)$ & $H C\left(1^{\prime}\right)$ \\
$\mathrm{HC}(4)$ & $\sim 2.7(\mathrm{~m})$ & $2.67(\mathrm{dtd}, J=13.5,7.5,1.0)$ & $\mathrm{HC}(4)$ \\
$\mathrm{HC}\left(1^{\prime}\right)$ & $\sim 2.60(\mathrm{dt}, J=14.0,8.0,8.0)$ & $2.61(\mathrm{dddd}, J=14.0,7.0,6.5,1.0)$ & $\mathrm{HC}\left(1^{\prime}\right)$ \\
$H C\left(1^{\prime}\right)$ & $2.50(\mathrm{ddd}, J=14.0,8.0,3.0)$ & $2.48(\mathrm{dd}, J=13.5,8.0)$ & $H C(4)$ \\
$\mathrm{HC}(10)$ & $1.73(\mathrm{dq}, J=14.0 .7 .0)$ & $1.72(\mathrm{~d} \mathrm{quint}, J=14.5,7.5)$ & $\mathrm{HC}(10)$ \\
$\mathrm{HC}(10)$ & $1.55(\mathrm{dq}, J=14.0 .7 .0)$ & $1.53(\mathrm{~d} \mathrm{quint}, J=14.5,7.5)$ & $\mathrm{HC}(10)$ \\
$\mathrm{HC}(11)$ & $0.91(\mathrm{t}, J=7.0)$ & $0.91(\mathrm{t}, J=7.5)$ & $\mathrm{HC}(11)$ \\
\hline
\end{tabular}


Table 2. ${ }^{13} \mathrm{C}$ NMR Data of Natural and Synthetic (+)-Brasilenyne (1)

\begin{tabular}{|cccc|}
\hline $\begin{array}{c}\text { Natural 1 } \\
\text { Solvent: } \mathrm{CCl}_{4}(220 \mathrm{MHz}) \\
\text { Ref. peak: TMS }\end{array}$ & $\begin{array}{c}\text { Synthetic 1 } \\
\text { Solvent: } \mathrm{CDCl}_{3}(500 \mathrm{MHz})\end{array}$ & $\begin{array}{c}\text { Assignment } \\
\text { (synthetic 1) }\end{array}$ & $\Delta^{13} \mathrm{C}$ \\
\hline 141.2 & 141.39 & $\mathrm{HC}\left(2^{\prime}\right)$ & +0.19 \\
134.2 & 134.34 & $\mathrm{HC}(8)$ & +0.14 \\
133.3 & 133.52 & $\mathrm{HC}(7)$ & +0.22 \\
129.5 & 129.65 & $\mathrm{HC}(6)$ & +0.15 \\
128.6 & 128.74 & $\mathrm{HC}(5)$ & +0.14 \\
110.4 & 110.56 & $\mathrm{HC}\left(3^{\prime}\right)$ & +0.16 \\
82.0 & 82.17 & $\mathrm{HC}\left(5^{\prime}\right)$ & +0.17 \\
80.1 & 80.20 & $\mathrm{HC}\left(4^{\prime}\right)$ & +0.10 \\
76.0 & 75.65 & $\mathrm{HC}(2)$ & -0.35 \\
75.6 & 75.54 & $\mathrm{HC}(9)$ & -0.06 \\
62.9 & 63.00 & $\mathrm{HC}(3)$ & +0.10 \\
36.3 & 36.41 & $\mathrm{HC}\left(1^{\prime}\right)$ & +0.11 \\
35.9 & 35.98 & $\mathrm{HC}(4)$ & +0.08 \\
28.5 & 28.61 & $\mathrm{HC}(10)$ & +0.11 \\
9.94 & 10.08 & $\mathrm{HC}(11)$ & +0.14 \\
\hline
\end{tabular}

\section{References}

(1) Gilman, H.; Cartledge, F. K.; Sin, S.-Y. J. Organomet. Chem. 1963, 1, 8.

(2) Chemistry of Metal-Carbon Bonds, Vol. 1, Patai and Hartley Ed. Chapter 156, p 639.

(3) The Schrock's molybdenum complex is commercially available (Strem) and can be prepared according to the reported procedure with consistent purity and reactivity, see: (1) Fox, H. H.; Yap, K. B.; Robbins, J.; Cai, S.; Schrock, R. R. Inorg. Chem. 1992, 31, 2287. (b) Schrock, R. R.; Murdzek, J. S.; Bazan, G. C.; Robbins, J.; DiMare, M.; O’Regan, M. J. Am. Chem. Soc. 1990, 112, 3875. (c) Oskam, J. H.; Fox, H. H.; Yap, K. B.; McConville, D. H.; O’Dell, R.; Lichtenstein, B. J.; Schrock, R. R. J. Organomet. Chem. 1993, 459, 185. (d) Fox, H. H.; Lee, J.-K.; Park, L. Y.; Schrock, R. R. Organometallics 1993, 12, 759.

(4) Kinnel, R.B.; Dieter, R. K.; Meinwald, J.; Engen, D. V.; Clardy, J. Eisner, T.; Stallard, M. O.; Fenical, W. Proc. Natl. Acad. Sci. USA 1979, 76, 3576. 

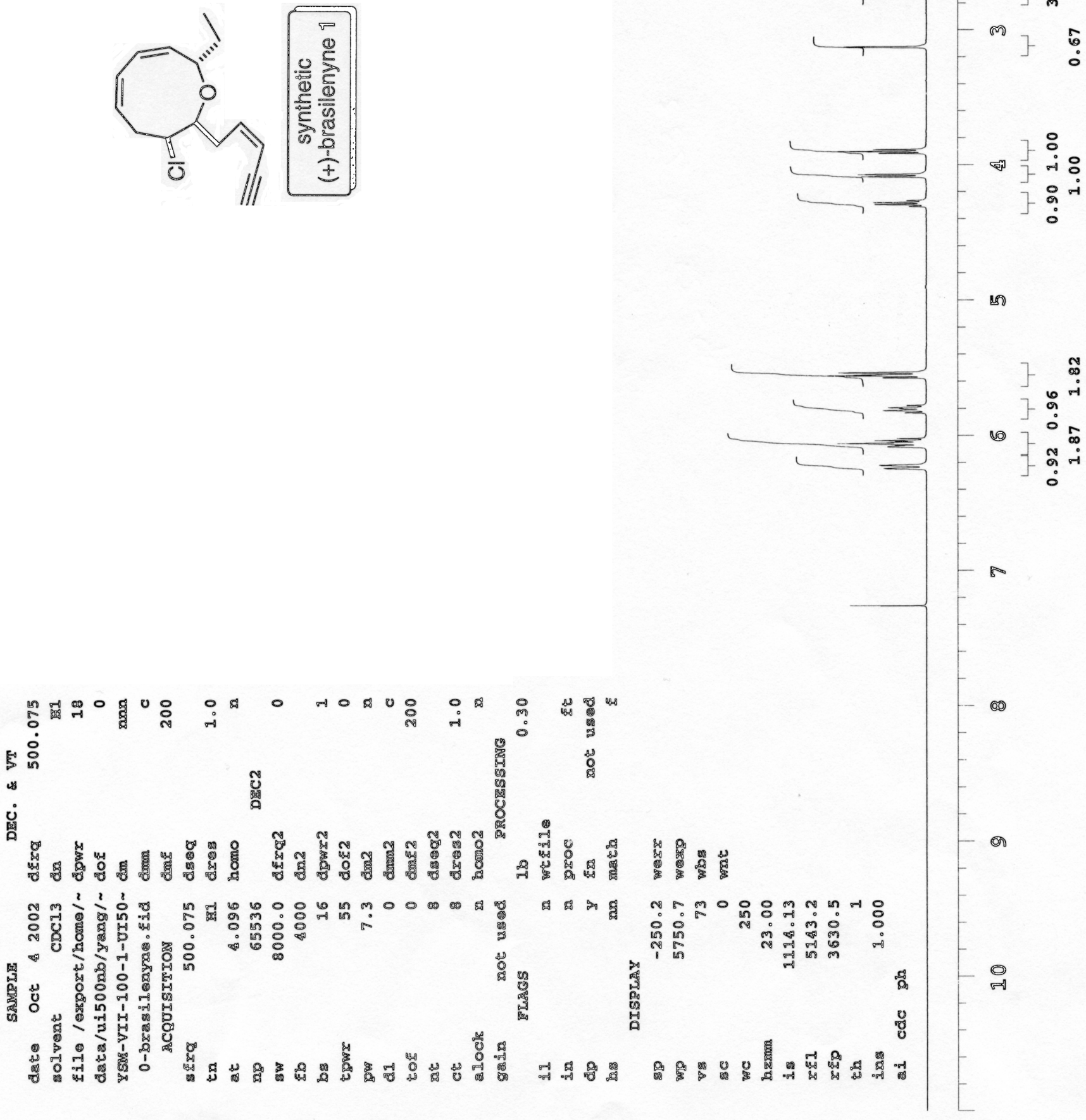

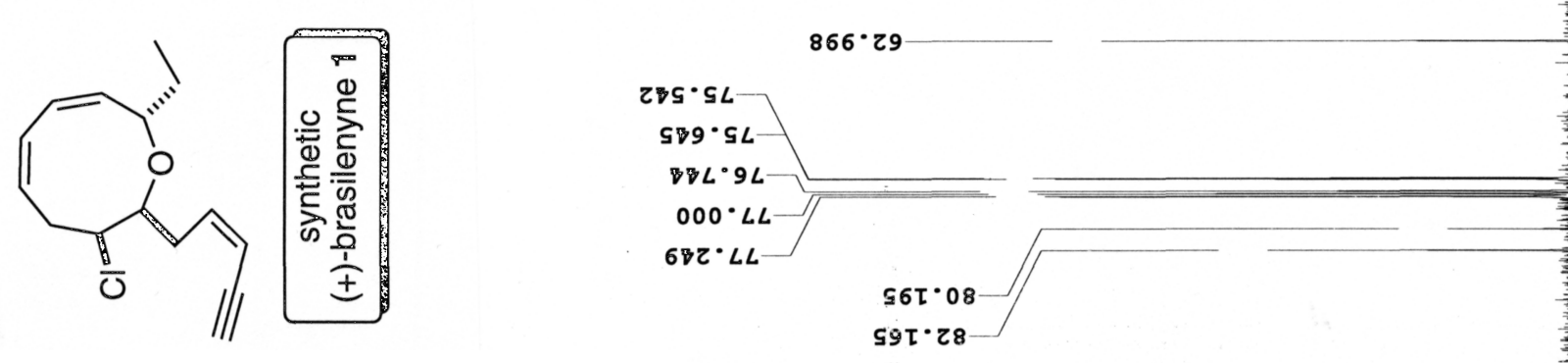

웅

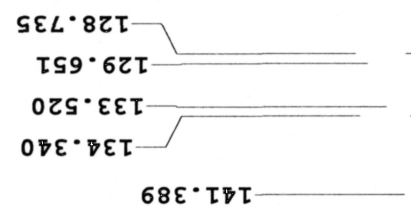

$\stackrel{\text { ㄱ }}{\text { r }}$

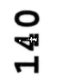

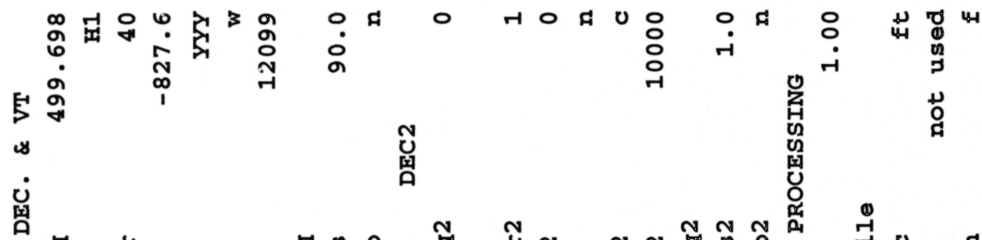

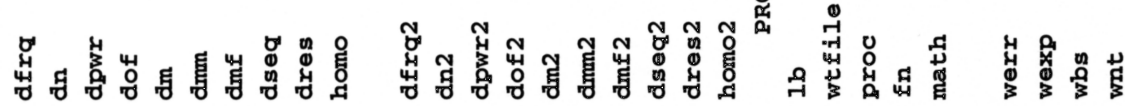

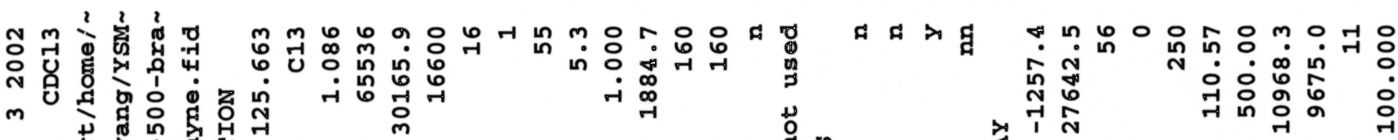

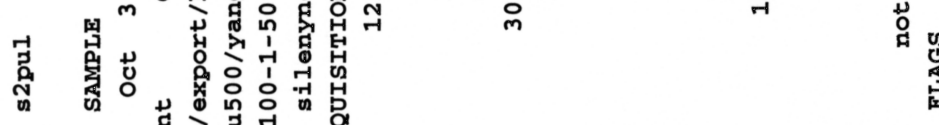

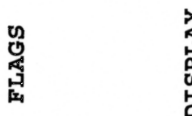

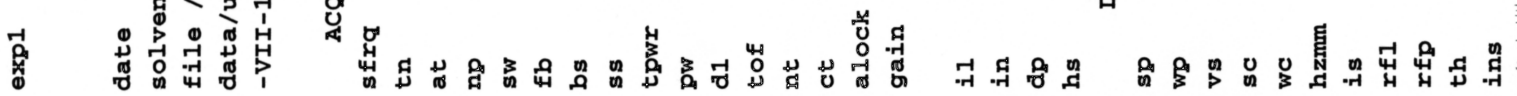




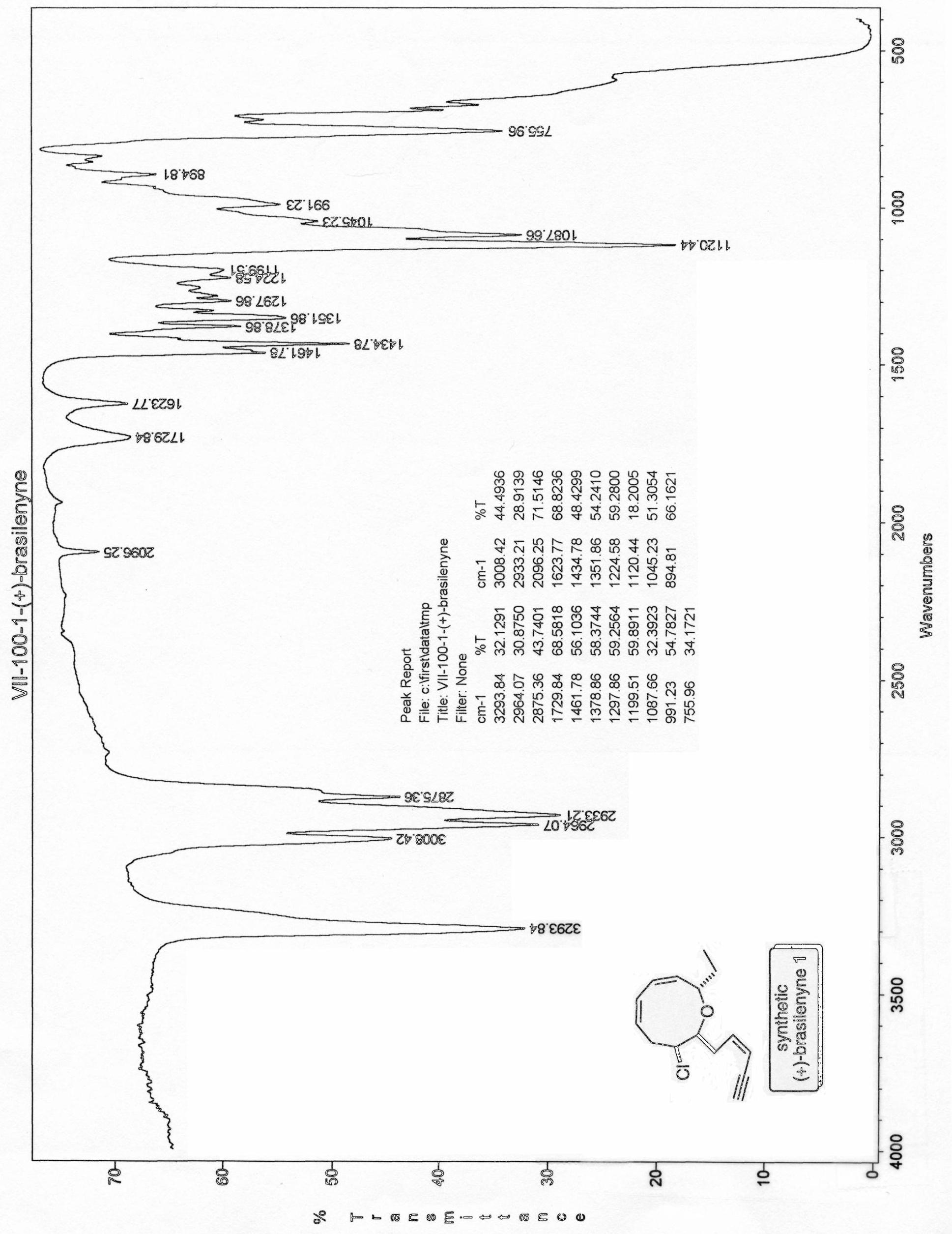

